\title{
The Monogenic Fischer Decomposition: Two Vector Variables
}

\author{
P. Van Lancker*
}

May 8, 2011

\begin{abstract}
In this paper we will present two proofs of the monogenic Fischer decomposition in two vector variables. The first one is based on the so-called "Harmonic Separation of Variables Theorem" while the second one relies on some simple dimension arguments. We also show that these decomposition are still valid under milder assumptions than the usual stable range condition. In the process, we derive explicit formula for the summands in the monogenic Fischer decomposition of harmonics.
\end{abstract}

\section{Introduction}

The so-called Separation of Variables Theorem is closely connected with the invariant theory of the classical groups. Roughly speaking, this theorem can be explained as follows (see [19]). Let $G$ be a reductive algebraic group acting on a vector space $W$ and extend this action to the algebra of polynomials $\mathcal{P}(W)$ on $W$. Denote the algebra of $G$-invariants in $\mathcal{P}(W)$ as $\mathcal{P}(W)^{G}$ and the algebra of $G$-invariant constant-coefficient partial differential operators on $W$ as $\mathcal{D}(W)^{G}$. Let $\operatorname{Ker}_{G}(W):=\left\{P \in \mathcal{P}(W): D P=0\right.$ for all $\left.D \in \mathcal{D}(W)^{G}\right\}$ be the space of $G$-harmonic polynomials. The Separation of Variables Theorem states that

$$
\mathcal{P}(W) \simeq \mathcal{P}(W)^{G} \otimes \operatorname{Ker}_{G}(W)
$$

within a certain stable range. In the framework of Clifford analysis, this theorem is usually referred to as the Fischer decomposition. In this paper we give two alternative proofs of the monogenic Fischer decomposition for polynomials of several vector variables in the easiest non-trivial case of two vector variables. This corresponds to the case where $G=\operatorname{Spin}(m)$ acts by the $L$-representation and $W=\mathbb{R}^{2 \times m}$. A more precise statement of the theorem will be postponed untill the necessary background has been treated in the introductory part.

Our approach aims to elucidate the interplay between different ingredients: the $L$-action of the group $\operatorname{Spin}(m)$, the orthosymplectic Lie (super) algebra osp $(1 \mid 2 k$ ) (both acting on Clifford algebra-valued polynomials of several vector variables) and monogenic polynomials of several vector variables. The study of this type of polynomials has its origins in the work [9] by D. Constales. We will discuss all this in detail for the case of two vector variables $(k=2)$.

*e-mail:Peter.VanLancker@hogent.be, Department of Engineering Sciences, University College Ghent, Member of Ghent University Association, Schoonmeersstraat 52, 9000 GENT (Belgium)

2010 Mathematics Subject Classification. 30G35, 42B37, 35J05.

Keywords. Clifford analysis, Dirac operators, Representations, Fischer decomposition, Spin groups. 
We would like to point out that some of the results we present here were already known and proved elsewhere. Our contribution rather lies in the fact that we provide alternative proofs and that we present our results as part of the general scheme of invariant theory as mentioned before. In particular, the well known results for the rotation group $\mathrm{SO}(m)$ are extended to its double cover $\operatorname{Spin}(m)$, thus offering the possibily to incorporate also the half-integer weight representations of $\operatorname{Spin}(m)$ into this picture. This is exactly where tools of Clifford analysis and in particular Dirac operators, turn out to be quite useful.

The monogenic (and harmonic) Fischer decomposition in several variables plays a fundamental role in recent work involving the representation theory of $\operatorname{Spin}(m)$, the Howe dual pairs $(\mathfrak{o s p}(1 \mid 2 k)$, Spin $(m))$ (see e.g. [23, 31]), syzygies and resolutions occurring in algebraic analysis of several Dirac operators (see e.g. [8,22]), the study of higher spin (Stein-Weiss) and RaritaSchwinger operators (see e.g. $[3,4,5,6,7,13,30]$ ).

The paper is organized as follows. In a preliminary section, we collect some basic notions about Clifford analysis and the invariant theory of $\mathrm{SO}(m)$ and $\operatorname{Spin}(m)$. Next, we recall some facts concerning the harmonic Fischer decomposition. A basic problem in Clifford analysis is the decomposition of harmonic polynomials into monogenics. We will determine the structure of this decomposition and show how the summands can be computed in an explicit way. Together with the harmonic Fischer decomposition, these are the key ingredients of our first proof of the monogenic Fischer decomposition in two vector variables (in a certain stable range). The advantage of this proof lies in the fact that it can generalized to the $k$-vector variable case. It also makes clear that monogenic polynomials of several vector variables are not a straightforward refinement of harmonic polynomials. Our second proof and the method used in [28] based on a dimension formula proved in [9], seem to be more restricted to the two-vector variable case. However, also this proof has its merits because it shows that the usual stable range conditions can be slightly weakened. Finally, we discuss so-called counterexamples to either the harmonic or monogenic Fischer decomposition in the non-stable range $m=2$. These examples can also be generalized to the $k$-vector variable case. To the best of our knowledge, such examples are usually missing in the literature.

\subsection{Clifford analysis}

In this section we collect some basic material concerning Clifford analysis. More detailed information on this subject can be found e.g. in [2],[12],[14],[16].

Let $\left(e_{1}, \ldots, e_{m}\right)$ be an orthonormal basis of Euclidean space $\mathbb{R}^{m}$ endowed with the inner product $\langle x, y\rangle=\sum_{i=1}^{m} x_{i} y_{i}, x, y \in \mathbb{R}^{m}$. By $\mathbb{R}_{0, m}$ we denote the real $2^{m}$-dimensional Clifford algebra over $\mathbb{R}^{m}$ generated by the relations

$$
e_{i} e_{j}+e_{j} e_{i}=-2 \delta_{i j} .
$$

If there is no confusion possible we use the shorter notation $\mathbb{R}_{m}:=\mathbb{R}_{0, m}$. An element of $\mathbb{R}_{m}$ is of the form $a=\sum_{A \subset M} a_{A} e_{A}, a_{A} \in \mathbb{R}, M=\{1, \ldots, m\}$ where $A$ is an ordered subset of $M$ and $e_{\phi}=e_{0}=1$. The $k$-vector part of $a$ is given by $[a]_{k}=\sum_{|A|=k} a_{A} e_{A}$ and $a=\sum_{k=0}^{m}[a]_{k}$ with $[a]_{k} \in \mathbb{R}_{m}^{k}$. Vectors $x \in \mathbb{R}^{m}$ are identified with 1 -vectors $\underline{x}=\sum_{j=1}^{m} x_{j} e_{j} \in \mathbb{R}_{m}^{1}$. The Clifford product of two vectors $x$ and $y$ splits into minus the inner product and the wedge product of $x$ and $u$ :

$$
\underline{x} \underline{u}=-\langle\underline{x}, \underline{u}\rangle+\underline{x} \wedge \underline{u} .
$$


The complex Clifford algebra $\mathbb{C}_{m}$ is the complexification of $\mathbb{R}_{m}$. The following (anti-)involutions are of importance. They are defined by their action on the basis elements $e_{i} \in \mathbb{R}_{m}^{1}$ and extended by linearity to $\mathbb{R}_{m}$ :

- main involution $a \mapsto a^{\prime} ; \quad(a c)^{\prime}=a^{\prime} c^{\prime}, \quad e_{i}^{\prime}=-e_{i}$,

- conjugation $a \mapsto \bar{a} ; \quad \overline{a c}=\bar{c} \bar{a}, \quad \bar{e}_{i}=-e_{i}$.

The main involution ' defines a $\mathbb{Z}_{2}$-grading on $\mathbb{R}_{m}$. The eigenspaces $\mathbb{R}_{m}^{ \pm}$of the main involution ' are the so-called even and odd part of the Clifford algebra. Conjugation on $\mathbb{C}_{m}$ is the antiinvolution on $\mathbb{C}_{m}$ given by $\bar{a}=\sum_{A \subset M} \bar{a}_{A} \bar{e}_{A}$.

The subset of $\mathbb{R}_{m}$ consisting of products of an even number of unit vectors in $\mathbb{R}^{m}$ (identified with $\left.\mathbb{R}_{m}^{1}\right)$ forms a group under Clifford multiplication, this is the Spin group $\operatorname{Spin}(m):=$ $\operatorname{Spin}(0, m)$. The action of $s \in \operatorname{Spin}(m)$ on vectors $\underline{x} \in \mathbb{R}^{m}$ is the (twisted) adjoint vector representation $\chi(s) x:=s^{\prime} \underline{x} s^{-1}=s \underline{x} s^{-1}$. The map $\chi: \operatorname{Spin}(m) \rightarrow \mathrm{SO}(m), s \mapsto \chi(s)$ defines a double covering of the orthogonal group $\mathrm{SO}(m)$. A spinor space $\mathbb{S} \subset \mathbb{C}_{m}$ is an irreducible representation of $\operatorname{Spin}(m)$ under the action $s \mapsto s a, a \in \mathbb{C}_{m}$ and can be realized as a minimal left ideal in $\mathbb{C}_{m}$ using a primitive idempotent.

The Dirac operator on $\mathbb{R}^{m}$ is given by $\underline{\partial}_{x}=\sum_{j=1}^{m} e_{j} \partial_{x_{j}}$. Let $V$ be a Clifford module (typically $\mathbb{R}_{m}, \mathbb{C}_{m}$ or a spinor space $\mathbb{S}$ ). A $V$-valued function $f(x)$ is called monogenic if it satisfies the equation $\underline{\partial}_{x} f=0$. Since $\underline{\partial}_{x}^{2}=-\triangle_{x}$, monogenic functions are a refinement of harmonic functions. The space of $V$-valued polynomials on $\mathbb{R}^{m}$ is denoted by $\mathcal{P}\left(\mathbb{R}^{m}, V\right)$ and

$$
\begin{aligned}
\mathcal{H}\left(\mathbb{R}^{m}, V\right) & =\left\{P \in \mathcal{P}\left(\mathbb{R}^{m}, V\right): \triangle_{x} P(x)=0\right\} \\
\mathcal{M}\left(\mathbb{R}^{m}, V\right) & =\left\{P \in \mathcal{P}\left(\mathbb{R}^{m}, V\right): \partial_{x} P(x)=0\right\} .
\end{aligned}
$$

The corresponding subspaces of $k$-homogeneous polynomials are denoted by an extra subscript $k$. The spaces $\mathcal{M}_{k}\left(\mathbb{R}^{m}, V\right)$ and $\mathcal{H}_{k}\left(\mathbb{R}^{m}, V\right)$ are known as the spaces of (inner) spherical monogenics and harmonics of order $k$. Further information on spherical monogenics can be found in e.g. $[2,12,16]$.

The Fischer decomposition is a useful property which is particularly well known in the one variable case. It describes how polynomials on $\mathbb{R}^{m}$ can be decomposed in a unique way into basic building blocks of harmonic or monogenic polynomials:

$$
\mathcal{P}\left(\mathbb{R}^{m}, \mathbb{C}\right)=\bigoplus_{s \geq 0}|\underline{x}|^{2 s} \mathcal{H}\left(\mathbb{R}^{m}, \mathbb{C}\right), \quad \mathcal{P}\left(\mathbb{R}^{m}, \mathbb{C}_{m}\right)=\bigoplus_{s \geq 0} \underline{x}^{s} \mathcal{M}\left(\mathbb{R}^{m}, \mathbb{C}_{m}\right) .
$$

The group action behind these decompositions is either the $h$ - or $L$-action of $\operatorname{Spin}(m)$. One can also consider other actions: e.g. the $H$-action on $\mathbb{C}_{m}$-valued polynomials (cf. infra). The decomposition of $\mathcal{P}\left(\mathbb{R}^{m}, \mathbb{C}_{m}\right)$ under this action has recently been studied in the paper [11]. In this case, the basic building blocks are the spaces of homogeneous solutions to the Hodge-de Rham system.

\subsection{Invariants for the $\mathrm{SO}(m)$ - and $\operatorname{Spin}(m)$-action}

Denote by $\mathcal{P}\left(\mathbb{R}^{m \times 2}, V\right)$ the space of $V$-valued polynomials on $\mathbb{R}^{m \times 2}$ where $V$ is a Clifford module. Introduce the vector variables

$$
\underline{x}=\sum_{i=1}^{m} x_{i} e_{i}, \quad \underline{u}=\sum_{i=1}^{m} u_{i} e_{i}
$$


Polynomials $P(x, u)=P\left(x_{1}, \ldots, x_{m}, u_{1}, \ldots, u_{m}\right) \in \mathcal{P}\left(\mathbb{R}^{m \times 2}, V\right)$ correspond to polynomials of two vector variables $(x, u) \in \mathbb{R}^{m} \oplus \mathbb{R}^{m} \simeq \mathbb{R}^{m \times 2}$. Let $s \in \operatorname{Spin}(m)$. Consider the following representations of $\operatorname{Spin}(m)$ on $\mathcal{P}\left(\mathbb{R}^{m \times 2}, V\right)$ :

$$
\begin{aligned}
(h(s) P)(x, u) & =P\left(s^{-1} x s, s^{-1} u s\right)=P(\bar{s} x s, \bar{s} u s) \quad(V=\mathbb{C}) \\
(H(s) P)(x, u) & =s P\left(s^{-1} x s, s^{-1} u s\right) s^{-1}=s P(\bar{s} u s, \bar{s} u s) \bar{s} \quad\left(V=\mathbb{C}_{m}\right) \\
(L(s) P)(x, u) & =s P\left(s^{-1} x s, s^{-1} u s\right)=s P(\bar{s} u s, \bar{s} u s) \quad\left(V=\mathbb{C}_{m} \text { or } \mathbb{S}\right) .
\end{aligned}
$$

Remark that $h$ and $H$ are also $\mathrm{SO}(m)$-representations. Define the spaces of polynomial invariants:

$$
\begin{aligned}
\mathcal{I}(\mathrm{SO}(m), 2) & :=\left\{P \in \mathcal{P}\left(\mathbb{R}^{m \times 2}, \mathbb{C}\right): h(s) P=P, s \in \mathrm{SO}(m)\right\} \\
\mathcal{I}(\operatorname{Spin}(m), 2) & :=\left\{P \in \mathcal{P}\left(\mathbb{R}^{m \times 2}, \mathbb{C}_{m}\right): H(s) P=P, s \in \operatorname{Spin}(m)\right\}
\end{aligned}
$$

The extra number 2 in the notation for the polynomial invariants $\mathcal{I}$ emphasizes that we consider polynomial invariants of two vector variables. The First Fundamental Theorem of Invariant Theory (FFT) for $\mathrm{SO}(m)$ goes back to $\mathrm{H}$. Weyl, [32] and describes a set of basic generators for the invariants of the action of $\mathrm{SO}(m)$ :

$$
\mathcal{I}(\mathrm{SO}(m), 2) \simeq\left\{\begin{array}{l}
\mathcal{P}\left(|\underline{x}|^{2},|\underline{u}|^{2},\langle\underline{x}, \underline{u}\rangle\right) \oplus\left(x_{1} u_{2}-x_{2} u_{1}\right) \mathcal{P}\left(|\underline{x}|^{2},|\underline{u}|^{2},\langle\underline{x}, \underline{u}\rangle\right), \quad m=2 \\
\mathcal{P}\left(|\underline{x}|^{2},|\underline{u}|^{2},\langle\underline{x}, \underline{u}\rangle\right), \quad m \geq 3 .
\end{array}\right.
$$

The case $m=2$ is rather special because the determinant yields an extra invariant polynomial $x_{1} u_{2}-x_{2} u_{1}$ satisfying $\left(x_{1} u_{2}-x_{2} u_{1}\right)^{2}=|\underline{x}|^{2}|\underline{u}|^{2}-\langle\underline{x}, \underline{u}\rangle^{2}$.

The algebra $\mathcal{I}(\operatorname{Spin}(m), 2)$ is more complicated. Let $\operatorname{Alg}(S)$ be the (unital) algebra over $\mathbb{C}$ generated by the elements in a set $S$. The algebra of $\operatorname{Spin}(m)$-invariant $\mathbb{C}_{m}$-valued polynomials is generated by the vector variables $\underline{x}, \underline{u}$ and the pseudoscalar $e_{M}=e_{12 \ldots m}$ (see [27]):

$$
\mathcal{I}(\operatorname{Spin}(m), 2) \simeq \operatorname{Alg}\left(\underline{x}, \underline{u}, e_{M}\right), \quad m \geq 2 .
$$

The generators of this algebra satisfy certain algebraic relations: the vectors $\underline{x}$ and $\underline{u}$ commute with the anti-commutator of two vectors and the pseudoscalar $e_{12 \ldots m}$ commutes $(m$ odd) or anti-commutes ( $m$ even) with the vector variables $\underline{x}$ and $\underline{u}$. In contrast with the $h$-action, the algebra $\operatorname{Alg}\left(\underline{x}, \underline{u}, e_{M}\right)$ does not behave like the symmetric algebra in the basic invariants and another type of algebra is needed. In fact, the subalgebra $\operatorname{Alg}\{\underline{x}, \underline{u}\}$ is a realization of the so-called radial algebra in two vector variables.

In case of $k$ vector variables, this algebra is defined as follows. Let $S=\left\{X_{1}, \ldots, X_{k}\right\}$ be a set of symbols, called abstract vector variables. Consider the free algebra $\mathcal{A}(S)$ over $\mathbb{C}$ generated by the set $S$. Let $I$ be the two-sided ideal in $\mathcal{A}(S)$ generated by the elements $\left[X_{i},\left\{X_{j}, X_{l}\right\}\right]$ where $(i, j, l)$ is any triple chosen from the set $\{1, \ldots, k\}$. The radial algebra of $k$ vector variables introduced in [25], is defined as

$$
\mathcal{R}(S)=\mathcal{R}\left(X_{1}, \ldots, X_{k}\right):=\mathcal{A}(S) / I .
$$

Consider the vector variables $\underline{x}_{j}=\sum_{s=1}^{m} x_{j s} e_{s}, j=1, \ldots, k$. Let $\operatorname{Alg}\left(\underline{x}_{1}, \ldots, \underline{x}_{k}\right)$ be the $\mathbb{C}$-algebra generated by these vector variables. The map $X_{j} \mapsto \underline{x}_{j}$ extends to an algebra isomorphism $\mathcal{R}\left(X_{1}, \ldots, X_{k}\right) \rightarrow \operatorname{Alg}\left(\underline{x}_{1}, \ldots, \underline{x}_{k}\right)$ iff $m \geq k$ (see [25]). In this paper, $k=2$ and $m \geq 2$. Hence, the algebra generated by the vector variables $\underline{x}$ and $\underline{u}$ is a realization of the 
radial algebra in two vector variables and we will often use the notation $\mathcal{R}(\underline{x}, \underline{u})$. We will also use the gradings

$$
\mathcal{I}(\mathrm{SO}(m))=\bigoplus_{l \geq 0} \mathcal{I}_{2 l}(\mathrm{SO}(m)), \quad \mathcal{R}(\underline{x}, \underline{u})=\bigoplus_{l \geq 0} \mathcal{R}_{l}(\underline{x}, \underline{u}) .
$$

where the subscript $j$ refers to the subspace of polynomials which are eigenfunctions of $E_{x}+E_{u}$ with eigenvalue $j$.

The radial algebra $\mathcal{R}(\underline{x}, \underline{u})$ admits a multivector structure which can be described as follows. Let $\wedge \mathfrak{f}_{-}$be the Grassmann algebra over the basic $\operatorname{Spin}(m)$-invariant vector variables $\underline{x}$ and $\underline{u}$ :

$$
\wedge \mathfrak{f}_{-} \simeq \mathbb{C} \oplus \mathbb{C} \underline{x} \oplus \mathbb{C} \underline{u} \oplus \mathbb{C}(\underline{x} \wedge \underline{u}) .
$$

One has the grading: $\wedge^{0} \mathfrak{f}_{-} \simeq \mathbb{C}, \wedge^{1} \mathfrak{f}_{-}=\mathfrak{f}_{-}=\operatorname{span}\{\underline{x}, \underline{u}\}, \wedge^{2} \mathfrak{f}_{-} \simeq \mathbb{C}(\underline{x} \wedge \underline{u})$ corresponding to scalars, vectors and bivectors. The map $a \mapsto[a]_{k}, a \in \mathbb{C}_{m}$ is $H$-invariant. Therefore one can also look for $k$-vector-valued $\operatorname{Spin}(m)$-invariant polynomials. As a consequence:

Lemma 1 The radial algebra (as a vector space) has the following multivector structure:

$$
\begin{aligned}
\mathcal{R}(\underline{x}, \underline{u}) & \simeq \mathcal{I}(\mathrm{SO}(m)) \otimes \wedge \mathfrak{f}_{-} \\
& \simeq \mathcal{P}\left(|\underline{x}|^{2},\langle\underline{x}, \underline{u}\rangle,|\underline{u}|^{2}\right) \otimes \wedge \mathfrak{f}_{-} .
\end{aligned}
$$

\subsection{The Lie (super)algebras $\mathfrak{s p}(4)$ and $\mathfrak{o s p}(1 \mid 4)$}

Let $P, Q \in \mathcal{P}\left(\mathbb{R}^{m \times 2}, \mathbb{C}_{m}\right)$. The Fischer inner product on $\mathcal{P}\left(\mathbb{R}^{m \times 2}, \mathbb{C}_{m}\right)$ is the positive definite Hermitean inner product defined as

$$
\langle P, Q\rangle:=\left.\left[\bar{P}\left(\partial_{x}, \partial_{u}\right) Q(x, u)\right]_{0}\right|_{x=0, u=0},
$$

where $P\left(\partial_{x}, \partial_{u}\right)$ stands for the constant coefficient partial differential operator obtained by replacing the coordinates $x_{i}, u_{i}$ by the corresponding derivatives $\partial_{x_{i}}, \partial_{u_{i}}$. The adjoint of $A \in \operatorname{End}\left(\mathcal{P}\left(\mathbb{R}^{m \times 2}, \mathbb{C}_{m}\right)\right)$ relative to the Fischer inner product is denoted by $A^{*}$. The coordinate variables $x_{i}, u_{i}$ and the derivatives $\partial_{x_{i}}, \partial_{u_{i}}$ are each others Fischer-adjoint while the generators $e_{i}$ of the Clifford algebra $\mathbb{C}_{m}$ are skew-adjoint. Therefore the Fischer-adjoints of the basis invariants $|\underline{x}|^{2},|\underline{u}|^{2},\langle\underline{x}, \underline{u}\rangle, \underline{x}, \underline{u}$ are given by the differential operators $\triangle_{x}, \triangle_{u}$, $\left\langle\underline{\partial}_{x}, \underline{\partial}_{u}\right\rangle,-\underline{\partial}_{x},-\underline{\partial}_{u}$. The Fischer-adjoint ${ }^{*}$ defines an involution between the algebra of $\mathrm{SO}(m)$ - or $\operatorname{Spin}(m)$-invariant polynomials (symbols) and the algebra of corresponding invariant constant-coefficient partial differential operators. Let $E_{x}=\left\langle\underline{x}, \underline{\partial}_{x}\right\rangle$ and $E_{u}=\left\langle\underline{u}, \underline{\partial}_{u}\right\rangle$ be the usual Euler operators. Define

$$
\begin{array}{rlrl}
\mathfrak{p}_{+} & =\operatorname{span}\left\{\triangle_{x}, \triangle_{u},\left\langle\underline{\partial}_{x}, \underline{\partial}_{u}\right\rangle\right\}, & & \mathfrak{p}_{-}=\operatorname{span}\left\{|\underline{x}|^{2},|\underline{u}|^{2},\langle\underline{x}, \underline{u}\rangle\right\}, \\
\mathfrak{f}_{+} & =\operatorname{span}\left\{\underline{\partial}_{x}, \underline{\partial}_{u}\right\}, & & \mathfrak{f}_{-}=\operatorname{span}\{\underline{x}, \underline{u}\} \\
\mathfrak{t} & =\operatorname{span}\left\{2 E_{x}+m, 2 E_{u}+m,\left\langle\underline{x}, \underline{\partial}_{u}\right\rangle,\left\langle\underline{u}, \underline{\partial}_{x}\right\rangle\right\} .
\end{array}
$$

Then $\mathfrak{s p}(4)=\mathfrak{p}_{-} \oplus \mathfrak{t} \oplus \mathfrak{p}_{+}$and $\mathfrak{o s p}(1 \mid 4)=\mathfrak{f}_{-} \oplus \mathfrak{f}_{+} \oplus \mathfrak{s p}(4)=\mathfrak{f}_{-} \oplus \mathfrak{p}_{-} \oplus \mathfrak{t} \oplus \mathfrak{p}_{+} \oplus \mathfrak{f}_{+}$. Here $\mathfrak{f}_{-} \oplus \mathfrak{f}_{+}$ stands for the odd (fermionic) part of the orthosymplectic Lie superalgebra osp $(1 \mid 4)$ while the symplectic Lie algebra $\mathfrak{s p}(4)$ is the even (bosonic) part. Lie superalgebras are $\mathbb{Z}_{2}$-graded algebras endowed with a non-associative operation defined by the Lie super-bracket. The Lie super-bracket of $X$ and $Y$ is defined as $X Y-(-1)^{|Y||X|} Y X$, where the grade of $|X|=0$ or 1 
if $X$ is even or odd. Commutators are denoted by $[X, Y]=X Y-Y X$ and anti-commutators by $\{X, Y\}=X Y+Y X$. The (anti-)commutation relations imply

$$
\begin{aligned}
& {\left[\mathfrak{p}_{+}, \mathfrak{p}_{-}\right]=\mathfrak{t}, \quad\left[\mathfrak{t}, \mathfrak{p}_{ \pm}\right]=\mathfrak{p}_{ \pm}, \quad\left\{\mathfrak{f}_{+}, \mathfrak{f}_{-}\right\}=\mathfrak{t}, \quad\left[\mathfrak{t}, \mathfrak{f}_{ \pm}\right]=\mathfrak{f}_{ \pm},} \\
& \left\{\mathfrak{f}_{+}, \mathfrak{f}_{+}\right\}=\mathfrak{p}_{+}, \quad\left\{\mathfrak{f}_{-}, \mathfrak{f}_{-}\right\}=\mathfrak{p}_{-}, \quad\left[\mathfrak{p}_{+}, \mathfrak{f}_{-}\right]=\mathfrak{f}_{+}, \quad\left[\mathfrak{p}_{-}, \mathfrak{f}_{+}\right]=\mathfrak{f}_{-}, \\
& {\left[\mathfrak{p}_{+}, \mathfrak{p}_{+}\right]=\left[\mathfrak{p}_{-}, \mathfrak{p}_{-}\right]=\left[\mathfrak{p}_{+}, \mathfrak{f}_{+}\right]=\left[\mathfrak{p}_{-}, \mathfrak{f}_{-}\right]=0 .}
\end{aligned}
$$

These relations can also be expressed in the universal enveloping algebra $U(\mathfrak{o s p}(1 \mid 4))$ as: $\mathfrak{p}_{+} \mathfrak{p}_{-} \subset \mathfrak{p}_{-} \mathfrak{p}_{+}+\mathfrak{t}, \mathfrak{f}_{+} \mathfrak{f}_{-} \subset \mathfrak{f}_{-} \mathfrak{f}_{+}+\mathfrak{t}$. By induction:

$$
\mathfrak{p}_{+}\left(\mathfrak{p}_{-}\right)^{j} \subset\left(\mathfrak{p}_{-}\right)^{j} \mathfrak{p}_{+}+\left(\mathfrak{p}_{-}\right)^{j-1}(\mathfrak{t}+1), \quad \mathfrak{f}_{+}\left(\mathfrak{f}_{-}\right)^{j} \subset\left(\mathfrak{f}_{-}\right)^{j} \mathfrak{f}_{+}+\left(\mathfrak{f}_{-}\right)^{j-1}(\mathfrak{t}+1) .
$$

\section{The harmonic Fischer decomposition}

Define the spaces of harmonic and monogenic polynomials in two vector variables:

$$
\begin{aligned}
\mathcal{H}\left(\mathbb{R}^{m \times 2}, \mathbb{C}\right) & =\left\{f \in \mathcal{P}\left(\mathbb{R}^{m \times 2}, \mathbb{C}\right): \triangle_{x} f=\triangle_{u} f=\left\langle\underline{\partial}_{x}, \underline{\partial}_{u}\right\rangle f=0 \text { or } \mathfrak{p}_{+} f=0\right\}, \\
\mathcal{M}\left(\mathbb{R}^{m \times 2}, V\right) & =\left\{f \in \mathcal{P}\left(\mathbb{R}^{m \times 2}, V\right): \underline{\partial}_{x} f=\underline{\partial}_{u} f=0 \text { or } \mathfrak{f}_{+} f=0\right\} .
\end{aligned}
$$

These spaces can alternatively be defined as the polynomials annihilated by all $\mathrm{SO}(m)$ - or $\operatorname{Spin}(m)$-invariant constant-coefficient differential operators of which the symbol vanishes in 0 . We have the following propositions. First of all, we formulate the so-called primitive Fischer decomposition which is valid in a wider range for $m$ than the usual Fischer decomposition. The former provides less detailed information and is easy to prove. Because of its similarity with the harmonic case, we mention here already the monogenic version as well.

Lemma 2 (Primitive harmonic and monogenic Fischer decomposition). Let $m \geq 2$. Then we have the orthogonal direct sum:

$$
\begin{aligned}
\mathcal{P}\left(\mathbb{R}^{m \times 2}, V\right) & =\mathcal{H}\left(\mathbb{R}^{m \times 2}, V\right) \oplus \mathfrak{p}_{-} \mathcal{P}\left(\mathbb{R}^{m \times 2}, V\right) \\
& =\bigoplus_{l \geq 0}\left(\mathfrak{p}_{-}\right)^{l} \mathcal{H}\left(\mathbb{R}^{m \times 2}, V\right) \\
\mathcal{P}\left(\mathbb{R}^{m \times 2}, V\right) & =\mathcal{M}\left(\mathbb{R}^{m \times 2}, V\right) \oplus\left(\underline{x} \mathcal{P}\left(\mathbb{R}^{m \times 2}, V\right)+\underline{u} \mathcal{P}\left(\mathbb{R}^{m \times 2}, V\right)\right) \\
& =\mathcal{M}\left(\mathbb{R}^{m \times 2}, V\right) \oplus \mathfrak{f}_{-} \mathcal{P}\left(\mathbb{R}^{m \times 2}, V\right) \\
& =\bigoplus_{l \geq 0}\left(\mathfrak{f}_{-}\right)^{l} \mathcal{M}\left(\mathbb{R}^{m \times 2}, V\right) .
\end{aligned}
$$

Proof.

To illustrate the idea, we only proof the monogenic version (5). The direct sum (4) is an immediate consequence of the fact that $\underline{x}$ an $\underline{u}$ are (up to a sign) the Fischer duals of $\underline{\partial}_{x}$ and $\underline{\partial}_{u}$. Repeated application of (4) shows that

$$
\mathcal{P}\left(\mathbb{R}^{m \times 2}, V\right)=\sum_{l \geq 0}\left(\mathfrak{f}_{-}\right)^{l} \mathcal{M}\left(\mathbb{R}^{m \times 2}, V\right) .
$$

Summands corresponding to different $l$ are Fischer orthogonal; this follows from the relations (3) in $U(\mathfrak{o s p}(1 \mid 4))$. Therefore the sum (6) is direct.

The structure of the vector spaces $\left(\mathfrak{p}_{-}\right)^{l} \mathcal{H}\left(\mathbb{R}^{m \times 2}, V\right), l \in \mathbb{N}$ follows from the standard Fischer decomposition which is only valid in a more restricted range for $m$. The following decomposition is precisely of the type as mentioned in the introduction. 
Theorem 3 (Harmonic Fischer decomposition in two vector variables) Let $m \geq 4$. Then:

$$
\begin{aligned}
\mathcal{P}\left(\mathbb{R}^{m \times 2}, \mathbb{C}\right) & \simeq \mathcal{I}(\mathrm{SO}(m), 2) \otimes \mathcal{H}\left(\mathbb{R}^{m \times 2}, \mathbb{C}\right) \\
& \simeq \mathcal{P}\left(|\underline{x}|^{2},|\underline{u}|^{2},\langle\underline{x}, \underline{u}\rangle\right) \otimes \mathcal{H}\left(\mathbb{R}^{m \times 2}, \mathbb{C}\right) \\
J H & \leftarrow J \otimes H .
\end{aligned}
$$

More information about this theorem and its assumptions on the number of (vector) variables and the dimension $m$ can be found in $[15,19]$. According to their results, the so-called stable range is the condition $\left[\frac{m}{2}\right] \geq 2$ (= number of vector variables) or $m \geq 4$. In this case, the map $J \otimes H \mapsto J H$ (the product of the polynomials $J$ and $H$ ) is injective. Therefore $\mathcal{P}\left(\mathbb{R}^{m \times 2}, \mathbb{C}\right)$ can be regarded as a free module over $\mathcal{I}(\mathrm{SO}(m), 2)$. The harmonic Fischer decomposition is in [19] also referred to as "Harmonic Separation of Variables Theorem". We would like to mention that the stable range condition can be relaxed to $m \geq 3$. We will return to this in the second proof of the monogenic Fischer decomposition (theorem 13).

The harmonic Fischer decomposition can be further refined by decomposing the harmonics into irreducible pieces for the $h$-action. These are harmonics which satisfy additional $\mathrm{SO}(m)$ invariant PDE coming from the $\mathfrak{t}$-subalgebra of $\mathfrak{s p}(4)$. We state here some definitions and results which will be used in the sequel. For convenience, we consider here also simplicial monogenics.

Definition 1 The spaces of simplicial harmonics [15, 19] and monogenics [23, 31] are:

$$
\begin{aligned}
\mathcal{S H}\left(\mathbb{R}^{2 \times m}, V\right) & :=\left\{H \in \mathcal{H}\left(\mathbb{R}^{2 \times m}, V\right):\left\langle\underline{x}, \underline{\partial}_{u}\right\rangle H=0\right\} \\
\mathcal{S} \mathcal{M}\left(\mathbb{R}^{2 \times m}, V\right) & :=\left\{M \in \mathcal{M}\left(\mathbb{R}^{2 \times m}, V\right):\left\langle\underline{x}, \underline{\partial}_{u}\right\rangle M=0\right\} .
\end{aligned}
$$

Consider the action of the Lie algebra $\mathfrak{s l}(2) \simeq \operatorname{span}\left\{\left\langle\underline{x}, \underline{\partial}_{u}\right\rangle,\left\langle\underline{u}, \underline{\partial}_{x}\right\rangle, E_{x}-E_{u}\right\}$ on $\mathcal{H}\left(\mathbb{R}^{2 \times m}, V\right)$ or $\mathcal{M}\left(\mathbb{R}^{2 \times m}, V\right)$ expressed by the commutation relations:

$$
\begin{aligned}
{\left[\left\langle\underline{x}, \underline{\partial}_{u}\right\rangle,\left\langle\underline{u}, \underline{\partial}_{x}\right\rangle\right] } & =E_{x}-E_{u} \\
{\left[E_{x}-E_{u},\left\langle\underline{x}, \underline{\partial}_{u}\right\rangle\right] } & =2\left\langle\underline{x}, \underline{\partial}_{u}\right\rangle \\
{\left[E_{x}-E_{u},\left\langle\underline{u}, \underline{\partial}_{x}\right\rangle\right] } & =-2\left\langle\underline{u}, \underline{\partial}_{x}\right\rangle .
\end{aligned}
$$

Under this action, harmonic (monogenic) polynomials can be further decomposed into simplicial harmonic (monogenic) polynomials. Notice that the operators $\left\langle\underline{x}, \underline{\partial}_{u}\right\rangle$ and $\left\langle\underline{u}, \underline{\partial}_{x}\right\rangle$ are each others Fischer-adjoint. This leads immediately to the direct sums:

$$
\mathcal{H}\left(\mathbb{R}^{2 \times m}, V\right)=\bigoplus_{j \geq 0}\left\langle\underline{u}, \underline{\partial}_{x}\right\rangle^{j} \mathcal{S H}\left(\mathbb{R}^{2 \times m}, V\right), \quad \mathcal{M}\left(\mathbb{R}^{2 \times m}, V\right)=\bigoplus_{j \geq 0}\left\langle\underline{u}, \underline{\partial}_{x}\right\rangle^{j} \mathcal{S} \mathcal{M}\left(\mathbb{R}^{2 \times m}, V\right) .
$$

The Casimir operator $C(\mathfrak{s l}(2))$ for this action is defined by:

$$
\begin{aligned}
C(\mathfrak{s l}(2)) & :=2\left(\left\langle\underline{x}, \underline{\partial}_{u}\right\rangle\left\langle\underline{u}, \underline{\partial}_{x}\right\rangle+\left\langle\underline{u}, \underline{\partial}_{x}\right\rangle\left\langle\underline{x}, \underline{\partial}_{u}\right\rangle\right)+\left(E_{x}-E_{u}\right)^{2} \\
& =4\left\langle\underline{u}, \underline{\partial}_{x}\right\rangle\left\langle\underline{x}, \underline{\partial}_{u}\right\rangle+\left(E_{x}-E_{u}\right)\left(E_{x}-E_{u}+2\right) .
\end{aligned}
$$

Lemma 4 Define the operator $G:=-4\left\langle\underline{u}, \underline{\partial}_{x}\right\rangle\left\langle\underline{x}, \underline{\partial}_{u}\right\rangle+\left(2 E_{x}+m\right)\left(2 E_{u}+m-2\right)$. Then:

1. $G=\left(E_{x}+E_{u}+m-1\right)^{2}-C(\mathfrak{s l}(2))-1$, hence $G$ belongs to the center of the universal enveloping algebra of $\mathfrak{g l}(2) \simeq \mathfrak{s l}(2) \oplus \mathbb{R}\left(E_{x}+E_{u}\right)$

2. $G$ defines an invertible endomorphism on harmonics and, in particular on monogenics

3. $\left(\underline{\partial}_{x} \wedge \underline{\partial}_{u}\right)((\underline{x} \wedge \underline{u}) P)=-\left(G+E_{x}+E_{u}+m\right) P, \quad P \in \mathcal{M}\left(\mathbb{R}^{2 \times m}, V\right)$. 
Proof.

(i) Follows from the definition of $G$ and expression (11).

(ii) First of all, $\left\langle\underline{u}, \underline{\partial}_{x}\right\rangle,\left\langle\underline{x}, \underline{\partial}_{x}\right\rangle, E_{x}, E_{u}$ define endomorphisms on either $\mathcal{M}\left(\mathbb{R}^{2 \times m}, V\right)$ or $\mathcal{H}\left(\mathbb{R}^{2 \times m}, V\right)$. To prove the invertibility, let $S_{k, l} \in \mathcal{S H}\left(\mathbb{R}^{2 \times m}, V\right)$ be homogeneous of bidegree $(k, l)$. Then $C(\mathfrak{s l}(2)) S_{k, l}=(k-l)(k-l+2) S_{k, l}$ and

$$
G S_{k, l}=(2 k+m)(2 l+m-2) S_{k, l} .
$$

This shows that $G$ is invertible on the space $\mathcal{S H}\left(\mathbb{R}^{2 \times m}, V\right)$. By $(i), G$ commutes with each power $\left\langle\underline{u}, \underline{\partial}_{x}\right\rangle^{j}$ in the decomposition (10). The smallest eigenvalue of $G$ (as an operator on $\left.\mathcal{H}\left(\mathbb{R}^{2 \times m}, V\right)\right)$ is $m(m+2)$, therefore $G$ is invertible on $\mathcal{H}\left(\mathbb{R}^{2 \times m}, V\right)$.

(iii) Consider the fundamental identity (see [24]):

$$
\begin{aligned}
& 2\left\{\Gamma_{x}, \Gamma_{u}\right\}+\left\{\underline{x} \wedge \underline{\partial}_{u}, \underline{u} \wedge \underline{\partial}_{x}\right\}+\left\{\underline{x} \wedge \underline{u}, \underline{\partial}_{x} \wedge \underline{\partial}_{u}\right\} \\
& +6 \triangle_{L B, x, u}-2(m-2)\left(\Gamma_{x}+\Gamma_{u}\right)+m(m-1)=0
\end{aligned}
$$

where $\triangle_{L B, x, u}:=\sum_{i<j} L_{x, i j} L_{u, i j}=\langle x, u\rangle\left\langle\partial_{x}, \partial_{u}\right\rangle-\left\langle u, \partial_{x}\right\rangle\left\langle x, \partial_{u}\right\rangle+E_{u}$ (see [31]). In particular for $P \in \mathcal{M}\left(\mathbb{R}^{2 \times m}, V\right)$ :

$$
\begin{aligned}
\triangle_{L B, x, u} P & =\left(-\left\langle\underline{u}, \underline{\partial}_{x}\right\rangle\left\langle\underline{x}, \underline{\partial}_{u}\right\rangle+E_{u}\right) P, \\
\left\{\underline{x} \wedge \underline{\partial}_{u}, \underline{u} \wedge \underline{\partial}_{x}\right\} P & =\left(\left\langle\underline{x}, \underline{\partial}_{u}\right\rangle\left\langle\underline{u}, \underline{\partial}_{x}\right\rangle+\left\langle\underline{u}, \underline{\partial}_{x}\right\rangle\left\langle\underline{x}, \underline{\partial}_{u}\right\rangle\right) P \\
& =\left(2\left\langle\underline{u}, \underline{\partial}_{x}\right\rangle\left\langle\underline{x}, \underline{\partial}_{u}\right\rangle+E_{x}-E_{u}\right) P, \\
\left\{\Gamma_{x}, \Gamma_{u}\right\} P & =2 E_{x} E_{u} P, \\
\left(\Gamma_{x}+\Gamma_{u}\right) P & =-\left(E_{x}+E_{u}\right) P .
\end{aligned}
$$

We thus obtain $\left(\underline{\partial}_{x} \wedge \underline{\partial}_{u}\right)((\underline{x} \wedge \underline{u}) P)=-\left(G+E_{x}+E_{u}+m\right) P$.

\section{The monogenic decomposition of harmonics}

In this section we determine the structure of the decomposition of harmonics into monogenics. In particular, we want to know how the space $\mathcal{M}\left(\mathbb{R}^{m \times 2}, V\right)$ can be embedded in a $\operatorname{Spin}(m)$ invariant way in $\mathcal{H}\left(\mathbb{R}^{m \times 2}, V\right)$. In the one-variable case this is quite simple:

$$
\left(1 \oplus \mathfrak{f}_{-}\right) \mathcal{M}\left(\mathbb{R}^{m}, V\right)=\mathcal{M}\left(\mathbb{R}^{m}, V\right) \oplus \underline{x} \mathcal{M}\left(\mathbb{R}^{m}, V\right)=\mathcal{H}\left(\mathbb{R}^{m}, V\right) .
$$

Only in this case, $\mathfrak{p}_{+}=\left(\mathfrak{f}_{+}\right)^{2}$. This fails to be true in the two-vector variable case because now $\mathfrak{p}_{+}$is not the full $\left(\mathfrak{f}_{+}\right)^{2}$ but rather the "symmetric or scalar part" of it. As a result, the monogenic Fischer decomposition in two vector variables is not a straightforward refinement of the harmonic Fischer decomposition. This property is also responsible for the fact that (although $\mathcal{M}\left(\mathbb{R}^{m \times 2}, V\right)$ is a subspace of $\left.\mathcal{H}\left(\mathbb{R}^{m \times 2}, V\right)\right)$ the monogenic decomposition of a harmonic polynomial will not be so simple. The following notion, based on the primitive Fischer decomposition, will play an important role:

Definition 2 The harmonic projection $P_{H}: \mathcal{P}\left(\mathbb{R}^{m \times 2}, \mathbb{C}\right) \rightarrow \mathcal{H}\left(\mathbb{R}^{m \times 2}, \mathbb{C}\right)$ is the projection on the first summand of the following orthogonal decomposition:

$$
\mathcal{P}\left(\mathbb{R}^{m \times 2}, \mathbb{C}\right)=\mathcal{H}\left(\mathbb{R}^{m \times 2}, \mathbb{C}\right) \oplus \mathfrak{p}_{-} \mathcal{P}\left(\mathbb{R}^{m \times 2}, \mathbb{C}\right) .
$$

We call $P_{H}[F]$ the harmonic part of $F$. 
In case of two (or more) vector variables one has to consider the harmonic projection $P_{H}$ acting on $\wedge \mathfrak{f}_{-} \mathcal{M}\left(\mathbb{R}^{m \times 2}, V\right)$. Clearly, $\left(1 \oplus \mathfrak{f}_{-}\right) \mathcal{M}\left(\mathbb{R}^{m \times 2}, V\right)$ is a strict subspace of $\mathcal{H}\left(\mathbb{R}^{m \times 2}, V\right)$. Finding the harmonic part of $(\underline{x} \wedge \underline{u}) \mathcal{M}\left(\mathbb{R}^{m \times 2}, V\right)$ is less straightforward. The following lemma provides an explicit formula.

Lemma 5 Let $m \geq 2$. Define $T_{1,1}:=|\underline{x}|^{2}\left\langle\underline{u}, \underline{\partial}_{x}\right\rangle-|\underline{u}|^{2}\left\langle\underline{x}, \underline{\partial}_{u}\right\rangle+\left(E_{u}-E_{x}\right)\langle\underline{x}, \underline{u}\rangle$. Then:

1. $T_{1,1}$ commutes with the $\mathfrak{s l}(2)$-action defined by the relations $(9)$

2. The harmonic projection of $(\underline{x} \wedge \underline{u}) M, M \in \mathcal{M}\left(\mathbb{R}^{m \times 2}, V\right)$ is given by:

$$
P_{H}[(\underline{x} \wedge \underline{u}) M]=\left(\underline{x} \wedge \underline{u}+\frac{1}{E_{x}+E_{u}+m-4} T_{1,1}\right) M
$$

3. $\left(\underline{\partial}_{x} \wedge \underline{\partial}_{u}\right)\left(T_{1,1} M\right)=C\left(\mathfrak{s l}_{2}\right) M, \quad M \in \mathcal{M}\left(\mathbb{R}^{m \times 2}, V\right)$.

Proof.

(i) Follows from standard computations in $\mathfrak{s p}(4)$.

(ii) Let $M:=M_{k, l} \in \mathcal{M}\left(\mathbb{R}^{m \times 2}, V\right)$ be homogeneous of bidegree $(k, l)$. Then $(\underline{x} \wedge \underline{u}) M_{k, l}$ is biharmonic, i.e. $(\underline{x} \wedge \underline{u}) M_{k, l}$ is annihilated by the ideal generated by $\mathfrak{p}_{+}^{2}$ where

$$
\mathfrak{p}_{+}^{2}=\operatorname{span}\left\{\triangle_{x}^{2}, \triangle_{u}^{2}, \triangle_{x} \triangle_{u}, \triangle_{x}\left\langle\underline{\partial}_{x}, \underline{\partial}_{u}\right\rangle, \triangle_{u}\left\langle\underline{\partial}_{x}, \underline{\partial}_{u}\right\rangle,\left\langle\underline{\partial}_{x}, \underline{\partial}_{u}\right\rangle^{2}\right\} .
$$

consists of all fourth-order $\mathrm{SO}(m)$-invariant constant coefficient differential operators. For $m \geq 2$ we have the $\mathbb{N}$-graded direct sum of lemma 2 :

$$
\mathcal{P}\left(\mathbb{R}^{m \times 2}, V\right)=\bigoplus_{l=0}^{\infty}\left(\mathfrak{p}_{-}\right)^{l} \mathcal{H}\left(\mathbb{R}^{m \times 2}, V\right) .
$$

If $\mathfrak{p}_{+}^{2} f=0$, then $f$ must be Fischer orthogonal to $\mathfrak{p}_{-}^{2} \mathcal{P}\left(\mathbb{R}^{m \times 2}, V\right)$. Hence $(\underline{x} \wedge \underline{u}) \mathcal{M}\left(\mathbb{R}^{m \times 2}, V\right) \subset$ $\mathcal{H}\left(\mathbb{R}^{m \times 2}, V\right) \oplus \mathfrak{p}_{-} \mathcal{H}\left(\mathbb{R}^{m \times 2}, V\right)$. Therefore we can take as Ansatz the following expression:

$$
H:=H_{k+1, l+1}=\left(\underline{x} \wedge \underline{u}+a|\underline{x}|^{2}\left\langle\underline{u}, \underline{\partial}_{x}\right\rangle+b|\underline{u}|^{2}\left\langle\underline{x}, \underline{\partial}_{u}\right\rangle+c\langle\underline{x}, \underline{u}\rangle\right) M_{k, l}
$$

and we will determine $a, b, c \in \mathbb{R}$ such that $H \in \mathcal{H}\left(\mathbb{R}^{m \times 2}, V\right)$. Remark that both $\left\langle\underline{u}, \underline{\partial}_{x}\right\rangle M_{k, l}$, $\left\langle\underline{x}, \underline{\partial}_{u}\right\rangle M_{k, l}$ belong to $\mathcal{M}\left(\mathbb{R}^{m \times 2}, V\right)$ because $\mathfrak{t}$ stabilizes $\mathcal{M}\left(\mathbb{R}^{m \times 2}, V\right)$. The action of $\triangle_{x}, \triangle_{u}$, $\left\langle\underline{\partial}_{x}, \underline{\partial}_{u}\right\rangle$ on $H$ yields a system of 3 equations in $a, b, c$ :

$$
\begin{aligned}
\triangle_{x} H= & -2\left\langle\underline{u}, \underline{\partial}_{x}\right\rangle M+2 a\left(2 E_{x}+m\right)\left\langle\underline{u}, \underline{\partial}_{x}\right\rangle M+2 c\left\langle\underline{u}, \underline{\partial}_{x}\right\rangle M \\
= & 2(c-1+(2 k+m-2) a)\left\langle\underline{u}, \underline{\partial}_{x}\right\rangle M=0 \\
\triangle_{u} H= & 2\left\langle\underline{x}, \underline{\partial}_{u}\right\rangle M+2 b\left(2 E_{u}+m\right)\left\langle\underline{x}, \underline{\partial}_{u}\right\rangle M+2 c\left\langle\underline{x}, \underline{\partial}_{u}\right\rangle M \\
= & 2(c+1+(2 l+m-2) b)\left\langle\underline{x}, \underline{\partial}_{u}\right\rangle M=0 \\
\left\langle\underline{\partial}_{u}, \underline{\partial}_{x}\right\rangle H= & 2\left(a\left\langle\underline{x}, \underline{\partial}_{u}\right\rangle\left\langle\underline{u}, \underline{\partial}_{x}\right\rangle+b\left\langle\underline{u}, \underline{\partial}_{x}\right\rangle\left\langle\underline{x}_{2}, \underline{\partial}_{u}\right\rangle\right) M \\
& +\left(E_{x}-E_{u}+c\left(m+E_{x}+E_{u}\right)\right) M=0 .
\end{aligned}
$$

The solution of this system is given by

$$
a=\frac{1}{k+l+m-2}, \quad b=-a, \quad c=\frac{l-k}{k+l+m-2} .
$$


(iii) Next, since $\left.\left[\underline{\partial}_{x} \wedge \underline{\partial}_{u}\right),|\underline{x}|^{2}\right]=2 \underline{x} \wedge \underline{\partial}_{u}$ and $\left[\underline{\partial}_{x} \wedge \underline{\partial}_{u},\langle\underline{u}, \underline{x}\rangle\right]=\Gamma_{x}-\Gamma_{u}$ :

$$
\begin{aligned}
\left(\underline{\partial}_{x} \wedge \underline{\partial}_{u}\right)|\underline{x}|^{2}\left\langle\underline{u}, \underline{\partial}_{x}\right\rangle M & =2\left\langle\underline{x}, \underline{\partial}_{u}\right\rangle\left\langle\underline{u}, \underline{\partial}_{x}\right\rangle M \\
\left(\underline{\partial}_{x} \wedge \underline{\partial}_{u}\right)|\underline{u}|^{2}\left\langle\underline{x}, \underline{\partial}_{u}\right\rangle M & =-2\left\langle\underline{u}, \underline{\partial}_{x}\right\rangle\left\langle\underline{x}, \underline{\partial}_{u}\right\rangle M \\
\left(\underline{\partial}_{x} \wedge \underline{\partial}_{u}\right)\left(E_{u}-E_{x}\right)\langle\underline{x}, \underline{u}\rangle M & =-\left(E_{u}-E_{x}\right)\left(\Gamma_{u}-\Gamma_{x}\right) M=\left(E_{x}-E_{u}\right)^{2} M .
\end{aligned}
$$

Combining all pieces yields

$$
\left(\underline{\partial}_{x} \wedge \underline{\partial}_{u}\right)\left(T_{1,1} M\right)=\left(4\left\langle\underline{u}, \underline{\partial}_{x}\right\rangle\left\langle\underline{x}, \underline{\partial}_{u}\right\rangle+\left(E_{x}-E_{u}\right)\left(E_{x}-E_{u}+2\right)\right) M=C\left(\mathfrak{s l}_{2}\right) M .
$$

The structure of the decomposition of harmonic polynomials into monogenic polynomials follows from:

Theorem 6 Suppose that $m \geq 3$ (weaker stable range).

1. Let $M \in \mathcal{M}\left(\mathbb{R}^{m \times 2}, V\right)$, then $: P_{H}[(\underline{x} \wedge \underline{u}) M]=0$ iff $M=0$

2. $\mathcal{H}\left(\mathbb{R}^{m \times 2}\right)=\mathcal{M}\left(\mathbb{R}^{m \times 2}\right) \oplus_{\perp}\left(\underline{x} \mathcal{M}\left(\mathbb{R}^{m \times 2}\right) \oplus \underline{u} \mathcal{M}\left(\mathbb{R}^{m \times 2}\right)\right) \oplus_{\perp} P_{H}\left[(\underline{x} \wedge \underline{u}) \mathcal{M}\left(\mathbb{R}^{m \times 2}\right)\right]$

where the subscript $\perp$ means orthogonal for the Fischer inner product.

Proof.

(i) Invoking the explicit form of the harmonic projection in lemma $5, P_{H}[(\underline{x} \wedge \underline{u}) M]=0$ if

$$
\left(E_{x}+E_{u}+m-2\right)(\underline{x} \wedge \underline{u}) M+T_{1,1} M=0 .
$$

Applying the operator $\left(\underline{\partial}_{x} \wedge \underline{\partial}_{u}\right)$ to this identity and using part (iii) of lemma 4 and 5 yields

$$
\left(E_{x}+E_{u}+m-1\right)\left(-\left(E_{x}+E_{u}+m\right)\left(E_{x}+E_{u}+m-2\right)+C\left(\mathfrak{s l}_{2}\right)\right) M=0 .
$$

Recall the decomposition of monogenics into simplicial monogenics (expression (10)). If we take $M=\left\langle\underline{u}, \underline{\partial}_{x}\right\rangle^{s} S_{k, l}, S_{k, l} \in \mathcal{S} \mathcal{M}\left(\mathbb{R}^{m \times 2}\right)$ in (13), we obtain

$$
-(k+l+m-1)(2 k+m)(2 l+m-2)\left\langle\underline{u}, \underline{\partial}_{x}\right\rangle^{s} S_{k, l}=0 .
$$

This is only possible if both $m=2$ and $l=0$ or $M=0$. This explains the condition $m \geq 3$. Remark that the counterexample (21) at the end of the paper is precisely an example where $m=2$ and $l=1$. We may thus conclude that for $m \geq 3$ the map $M \mapsto P_{H}[(\underline{x} \wedge \underline{u}) M]$, $\mathcal{M}\left(\mathbb{R}^{m \times 2}, V\right) \rightarrow \mathcal{H}\left(\mathbb{R}^{m \times 2}, V\right)$ defines a $\operatorname{Spin}(m)$-invariant embedding.

(ii) The summands in the primitive Fischer decomposition (5):

$$
\mathcal{P}\left(\mathbb{R}^{m \times 2}\right)=\bigoplus_{l \geq 0}\left(\mathfrak{f}_{-}\right)^{l} \mathcal{M}\left(\mathbb{R}^{m \times 2}\right)
$$

are Fischer orthogonal to each other. Since $P_{H}\left[(\underline{x} \wedge \underline{u}) \mathcal{M}\left(\mathbb{R}^{m \times 2}\right)\right] \in \mathfrak{f}_{-}^{2} \mathcal{M}\left(\mathbb{R}^{m \times 2}\right)$ :

$$
\mathcal{H}\left(\mathbb{R}^{m \times 2}\right)=P_{H}\left[\mathcal{P}\left(\mathbb{R}^{m \times 2}\right)\right]=\mathcal{M}\left(\mathbb{R}^{m \times 2}\right) \oplus_{\perp} \mathfrak{f}_{-} \mathcal{M}\left(\mathbb{R}^{m \times 2}\right) \oplus_{\perp} P_{H}\left[(\underline{x} \wedge \underline{u}) \mathcal{M}\left(\mathbb{R}^{m \times 2}\right)\right] .
$$


If $m \geq 3$ and $\underline{x} P+\underline{u} Q=0$ for some $P, Q \in \mathcal{M}\left(\mathbb{R}^{m \times 2}\right)$, then $|\underline{x}|^{2} P+\underline{x} \underline{u} Q=0$ which implies $P_{H}[(\underline{x} \wedge \underline{u}) Q]=0$. By part (i), $Q$ and hence also $P$ must be zero. Therefore, the second summand admits a direct sum decomposition:

$$
\mathfrak{f}_{-} \mathcal{M}\left(\mathbb{R}^{m \times 2}\right)=\underline{x} \mathcal{M}\left(\mathbb{R}^{m \times 2}\right)+\underline{u} \mathcal{M}\left(\mathbb{R}^{m \times 2}\right) \simeq \underline{x} \mathcal{M}\left(\mathbb{R}^{m \times 2}\right) \oplus \underline{u} \mathcal{M}\left(\mathbb{R}^{m \times 2}\right) .
$$

For applications it is of some interest to have explicit formula to determine all pieces in the monogenic Fischer decomposition of a harmonic polynomial. We will now show how these monogenic summands can be computed in a systematic way. First of all we state some definitions and results which will be used in the sequel.

Definition 3 Consider the following $\operatorname{Spin}(m)$-invariant operators:

$$
\begin{aligned}
& I_{-1,0}:=2\left\langle\underline{u}, \underline{\partial}_{x}\right\rangle \underline{\partial}_{u}-\left(2 E_{u}+m-2\right) \underline{\partial}_{x}, \\
& I_{0,-1}:=2\left\langle\underline{x}, \underline{\partial}_{u}\right\rangle \underline{\partial}_{x}-\left(2 E_{x}+m-2\right) \underline{\partial}_{u} .
\end{aligned}
$$

In the following lemma we collect some useful results concerning these operators.

Lemma 7 Let $m \geq 2$.

1. The operators $I_{-1,0}$ and $I_{0,-1}$ satisfy the anti-commutation relations:

$$
\begin{aligned}
& \left\{\underline{x}, I_{0,-1}\right\}=2 \underline{x} \underline{\partial}_{u}, \quad\left\{\underline{u}, I_{0,-1}\right\}=-2 \underline{x} \underline{\partial}_{x}+G, \\
& \left\{\underline{u}, I_{-1,0}\right\}=2 \underline{u}_{x}, \quad\left\{\underline{x}, I_{-1,0}\right\}=-2 \underline{\partial}_{u}+G
\end{aligned}
$$

and they map the kernel of $\left(\mathfrak{f}_{+}\right)^{2}$ to $\mathcal{M}\left(\mathbb{R}^{2 \times m}, V\right)$. In fact:

$$
\begin{aligned}
I_{-1,0}\left(M^{0,0}+\underline{u} M^{0,1}+\underline{x} M^{1,0}\right) & =G M^{1,0}, \\
I_{0,-1}\left(M^{0,0}+\underline{u} M^{0,1}+\underline{x} M^{1,0}\right) & =G M^{0,1}
\end{aligned}
$$

2. The operator $\underline{\partial}_{x} \wedge \underline{\partial}_{u}$ has a trivial kernel on $P_{H}\left[(\underline{x} \wedge \underline{u}) \mathcal{M}\left(\mathbb{R}^{2 \times m}, V\right)\right]$.

Proof.

(i) Straightforward computations yield the anti-commutation relations (valid on $\mathcal{P}\left(\mathbb{R}^{2 \times m}, V\right)$ ):

$$
\begin{aligned}
& \left\{\underline{x}, 2\left\langle\underline{x}, \underline{\partial}_{u}\right\rangle \underline{\partial}_{x}-\left(2 E_{x}+m-2\right) \underline{\partial}_{u}\right\}=2 \underline{x}_{u}, \\
& \left\{\underline{u}, 2\left\langle\underline{x}, \underline{\partial}_{u}\right\rangle \underline{\partial}_{x}-\left(2 E_{x}+m-2\right) \underline{\partial}_{u}\right\}=-2 \underline{x}_{x}+G
\end{aligned}
$$

and the similar relations with $\underline{x} \leftrightarrow \underline{u}(G$ is invariant under $\underline{x} \leftrightarrow \underline{u})$ :

$$
\begin{aligned}
& \left\{\underline{u}, 2\left\langle\underline{u}, \underline{\partial}_{x}\right\rangle \underline{\partial}_{u}-\left(2 E_{u}+m-2\right) \underline{\partial}_{x}\right\}=2 \underline{u}_{x}, \\
& \left\{\underline{x}, 2\left\langle\underline{u}, \underline{\partial}_{x}\right\rangle \underline{\partial}_{u}-\left(2 E_{u}+m-2\right) \underline{\partial}_{x}\right\}=-2 \underline{u}_{u}+G .
\end{aligned}
$$

The kernel of $\left(\mathfrak{f}_{+}\right)^{2}$ is $\mathcal{M}\left(\mathbb{R}^{2 \times m}\right) \oplus\left(\underline{u} \mathcal{M}\left(\mathbb{R}^{2 \times m}\right)+\underline{x} \mathcal{M}\left(\mathbb{R}^{2 \times m}\right)\right)$; the identities (14) follow immediately from the afore-mentioned anti-commutation relations .

(ii) Using part (iii) of lemma 4,5 and the explicit form of $P_{H}[(\underline{x} \wedge \underline{u}) P]$, we obtain

$$
\left(\underline{\partial}_{x} \wedge \underline{\partial}_{u}\right) P_{H}[(\underline{x} \wedge \underline{u}) P]=-\frac{G\left(E_{x}+E_{u}+m-1\right)}{E_{x}+E_{u}+m-2} P
$$

where the coefficient of $P$ is an invertible operator on $\mathcal{M}\left(\mathbb{R}^{2 \times m}, V\right)$. 
Theorem 8 (Monogenic decomposition of harmonics)

The summands in the decomposition

$$
H=M^{0,0}+\underline{x} M^{1,0}+\underline{u} M^{0,1}+P_{H}\left[(\underline{x} \wedge \underline{u}) M^{1,1}\right], \quad M^{a, b} \in \mathcal{M}\left(\mathbb{R}^{2 \times m}, V\right)
$$

can be determined recursively by:

$$
\begin{aligned}
P_{H}\left[(\underline{x} \wedge \underline{u}) M^{1,1}\right] & =B P_{H}\left[(\underline{x} \wedge \underline{u})\left(\underline{\partial}_{x} \wedge \underline{\partial}_{u}\right) H\right], \\
M^{1,0} & =G^{-1} I_{-1,0}\left(H-B P_{H}\left[(\underline{x} \wedge \underline{u})\left(\underline{\partial}_{x} \wedge \underline{\partial}_{u}\right) H\right]\right), \\
M^{0,1} & =G^{-1} I_{0,-1}\left(H-B P_{H}\left[(\underline{x} \wedge \underline{u})\left(\underline{\partial}_{x} \wedge \underline{\partial}_{u}\right) H\right]\right), \\
M^{0,0} & =H-\underline{x} M^{1,0}-\underline{u} M^{0,1}-P_{H}\left[(\underline{x} \wedge \underline{u}) M^{1,1}\right] .
\end{aligned}
$$

where $B:=-\frac{E_{x}+E_{u}+m-2}{G\left(E_{x}+E_{u}+m-1\right)}$.

Proof.

Recall the decomposition from Theorem 6:

$$
H=M^{0,0}+\underline{x} M^{1,0}+\underline{u} M^{0,1}+P_{H}\left[(\underline{x} \wedge \underline{u}) M^{1,1}\right], \quad M^{a, b} \in \mathcal{M}\left(\mathbb{R}^{2 \times m}, V\right) .
$$

To determine all summands we will first determine $M^{1,1}$ and work our way down to determine the other ones by recursion. By the previous lemma and relation (15), the operator $\underline{\partial}_{x} \wedge \underline{\partial}_{u}$ annihilates the first three terms in (16), hence

$$
\left(\underline{\partial}_{x} \wedge \underline{\partial}_{u}\right) H=\left(\underline{\partial}_{x} \wedge \underline{\partial}_{u}\right) P_{H}\left[(\underline{x} \wedge \underline{u}) M^{1,1}\right]=\frac{G\left(E_{x}+E_{u}+m-1\right)}{E_{x}+E_{u}+m-2} M^{1,1}=B M^{1,1} .
$$

Like the operator $G, B$ is also invertible on $\mathcal{M}\left(\mathbb{R}^{2 \times m}, V\right)$. Hence

$$
M^{1,1}=-\frac{E_{x}+E_{u}+m-2}{G\left(E_{x}+E_{u}+m-1\right)}\left(\underline{\partial}_{x} \wedge \underline{\partial}_{u}\right) H .
$$

To determine $M^{0,1}$ and $M^{1,0}$ we replace $H$ by $H^{\prime}=H-P_{H}\left[(\underline{x} \wedge \underline{u}) M^{1,1}\right]$. By relation (14) in the previous lemma: $I_{-1,0} H^{\prime}=G M^{1,0}$ and $I_{0,-1} H^{\prime}=G M^{0,1}$. We thus obtain

$$
\begin{aligned}
& M^{1,0}=G^{-1} I_{-1,0} H^{\prime}, \\
& M^{0,1}=G^{-1} I_{0,-1} H^{\prime}, \\
& M^{0,0}=H^{\prime}-\underline{x} M^{1,0}-\underline{u} M^{0,1} .
\end{aligned}
$$

\section{Monogenic polynomials in two vector variables}

Let $V=\mathbb{C}_{m}$ or $\mathbb{S}$. Denote by $\mathcal{M}_{l, k}\left(\mathbb{R}^{m \times 2}, V\right)$ the space of monogenic polynomials of bidegree $(k, l)$. We now want to calculate the dimension of the space $\mathcal{M}_{l, k}\left(\mathbb{R}^{m \times 2}, V\right)$. Our approach is based on the isomorphism given by the Cauchy-Kovalevska extension, thus providing an alternative approach to the one elaborated in [9]. In this section, we assume $m \geq 3$.

Put $\mathbb{R}^{m}=\mathbb{R} e_{1} \oplus e_{1}^{\perp}$ and identify $e_{1}^{\perp}$ with $\mathbb{R}^{m-1}$, we then use the notation $\underline{u}=u_{1} e_{1}+\underline{u}_{b}$ and $\underline{x}=x_{1} e_{1}+\underline{x}_{b}$. Restrict $P(x, u) \in \mathcal{M}\left(\mathbb{R}^{m \times 2}, V\right)$ to the plane $x_{1}=u_{1}=0$. This gives a polynomial $p\left(\underline{x}_{b}, \underline{u}_{b}\right)=P\left(0, \underline{x}_{b}, 0, \underline{u}_{b}\right) \in \mathcal{P}\left(\mathbb{R}^{m-1} \times \mathbb{R}^{m-1}, V\right)$. In contrast with the one-variable case, the polynomial $p\left(\underline{x}_{b}, \underline{u}_{b}\right)$ must also satisfy an equation (see also [9]): 
Theorem 9 The restriction $p\left(\underline{x}_{b}, \underline{u}_{b}\right)$ of $P(x, u) \in \mathcal{M}\left(\mathbb{R}^{m \times 2}, V\right)$ to the plane $x_{1}=u_{1}=0$ satisfies the PDE: $\left(\partial_{\underline{x}_{b}} \wedge \partial_{\underline{u}_{b}}\right) p\left(\underline{x}_{b}, \underline{u}_{b}\right)=0$ on $\mathbb{R}^{m-1} \times \mathbb{R}^{m-1}$. Moreover, $P(x, u)$ can be recovered from the (double) $\mathrm{CK}$-extension:

$$
P(x, u)=\exp \left(e_{1} u_{1} \partial_{\underline{u}_{b}}\right) \exp \left(e_{1} x_{1} \partial_{\underline{x}_{b}}\right) p\left(\underline{x}_{b}, \underline{u}_{b}\right)
$$

where the order of the operators defined by the exponentials is immaterial.

Definition 4 The Cauchy-Kovalevska extension $\mathrm{CK}$ for $\mathcal{M}\left(\mathbb{R}^{m \times 2}, V\right)$ is the isomorphism:

$$
\begin{aligned}
\mathrm{CK}: & \operatorname{Ker}\left(\partial_{\underline{x}_{b}} \wedge \partial_{\underline{u}_{b}}\right) \cap \mathcal{P}\left(\mathbb{R}^{m-1} \times \mathbb{R}^{m-1}, V\right) \rightarrow \mathcal{M}\left(\mathbb{R}^{m \times 2}, V\right) \\
& p\left(\underline{x}_{b}, \underline{u}_{b}\right) \mapsto P(x, u)=\exp \left(e_{1} x_{1} \partial_{\underline{x}_{b}}+e_{1} u_{1} \partial_{\underline{u}_{b}}\right) p\left(\underline{x}_{b}, \underline{u}_{b}\right) .
\end{aligned}
$$

Here, the key property of the CK-extension is that the system $\partial_{x} P=\partial_{u} P=0$ is replaced by a single equation $\left(\partial_{\underline{x}_{b}} \wedge \partial_{\underline{u}_{b}}\right) p\left(\underline{x}_{b}, \underline{u}_{b}\right)=0$. This is the main idea behind the following result:

Lemma 10 (Dimension of the space $\mathcal{M}_{l, k}\left(\mathbb{R}^{m \times 2}, V\right), m \geq 3$ )

$$
\begin{aligned}
\operatorname{dim} \mathcal{M}_{l, k}\left(\mathbb{R}^{m \times 2}, V\right) & =\operatorname{dim} \mathcal{P}_{l, k}\left(\mathbb{R}^{m-1} \times \mathbb{R}^{m-1}, V\right)-\operatorname{dim} \mathcal{P}_{l-1, k-1}\left(\mathbb{R}^{m-1} \times \mathbb{R}^{m-1}, V\right) \\
& =\left(\begin{array}{c}
k+m-3 \\
k-1
\end{array}\right)\left(\begin{array}{c}
l+m-3 \\
l-1
\end{array}\right) \frac{(k+l+m-2)(m-2)}{k l} \operatorname{dim} V
\end{aligned}
$$

Proof.

The Fischer dual of the operator $\partial_{\underline{x}_{b}} \wedge \partial_{\underline{u}_{b}}$ is given by the multiplication operator $\underline{u}_{b} \wedge \underline{x}_{b}$. We thus have a (primitive) Fischer orthogonal decomposition of the form:

$$
\mathcal{P}\left(\mathbb{R}^{m-1} \times \mathbb{R}^{m-1}, V\right)=\operatorname{Ker}\left(\partial_{\underline{x}_{b}} \wedge \partial_{\underline{u}_{b}}\right) \oplus\left(\underline{x}_{b} \wedge \underline{u}_{b}\right) \mathcal{P}\left(\mathbb{R}^{m-1} \times \mathbb{R}^{m-1}, V\right) .
$$

The condition $m \geq 3$ is needed because $\underline{x}_{b} \wedge \underline{u}_{b}$ is identically zero for $m=2$. We claim that the multiplication operator $\underline{u}_{b} \wedge \underline{x}_{b}$ is invertible on polynomials. Take a polynomial $Q$ such that $\left(\underline{x}_{b} \wedge \underline{u}_{b}\right) Q=0$. Then also $\left(\underline{x}_{b} \wedge \underline{u}_{b}\right)^{2} Q=\left(\left\langle\underline{x}_{b}, \underline{u}_{b}\right\rangle^{2}-\left|\underline{x}_{b}\right|^{2}\left|\underline{u}_{b}\right|^{2}\right) Q=0$, hence $Q=0$. The dimension formula then follows from $\operatorname{dim} \mathcal{P}_{k}\left(\mathbb{R}^{m}, V\right)=\left(\begin{array}{c}k+m-1 \\ k\end{array}\right) \operatorname{dim} V$.

\section{The monogenic Fischer decomposition}

The main result we want to prove is the analogue of the "Harmonic Separation of Variables Theorem" in the monogenic setting.

Theorem 11 (Monogenic Fischer decomposition in two vector variables) Let $m \geq 4$ and let $V$ be the Clifford algebra $\mathbb{C}_{m}$ or a spinor space $\mathbb{S}$. Then:

$$
\mathcal{P}\left(\mathbb{R}^{m \times 2}, V\right) \simeq \mathcal{R}(\underline{x}, \underline{u}) \otimes \mathcal{M}\left(\mathbb{R}^{m \times 2}, V\right) .
$$

The isomorphism is given by the map $J \otimes M \mapsto J M$, the invariant $J$ acting by left (Clifford) multiplication on $M$. Just as in the harmonic case, the map $J \otimes M \mapsto J M$ is injective, meaning that $\mathcal{P}\left(\mathbb{R}^{m \times 2}, V\right)$ can be regarded as a free (left) module over the radial algebra $\mathcal{R}(\underline{x}, \underline{u})$. 
Proof.

We use the following short notations: $\mathcal{M}:=\mathcal{M}\left(\mathbb{R}^{m \times 2}, V\right), \mathcal{H}:=\mathcal{H}\left(\mathbb{R}^{m \times 2}, V\right), \mathcal{I}_{2 j}:=$ $\mathcal{I}_{2 j}(\mathrm{SO}(m)), L_{l}:=\left(\mathfrak{f}_{-}\right)^{l} \mathcal{M}$. We already know that

$$
\mathcal{P}\left(\mathbb{R}^{m \times 2}, V\right)=\bigoplus_{l \geq 0}\left(\mathfrak{f}_{-}\right)^{l} \mathcal{M}\left(\mathbb{R}^{m \times 2}, V\right)=\bigoplus_{l \geq 0} L_{l} .
$$

We will now provide a basis for each layer $L_{l}$, considered as a module over $\left(\mathfrak{f}_{-}\right)^{l}$.

First of all, $L_{0}=\mathcal{M}$. Consider $L_{1}=\mathfrak{f}_{-} \mathcal{M}$. If $\underline{x} P+\underline{u} Q=0$ for $P, Q \in L_{0}$, then $|\underline{x}|^{2} P=\underline{x} \underline{u} Q$ and $P_{H}[\underline{x} \underline{u} Q]=P_{H}[(\underline{x} \wedge \underline{u}) Q]=0$. By lemma $5, Q=0$ and $P=0$, which shows that $L_{1} \simeq \underline{x} L_{0} \oplus \underline{u} L_{0}$ is a direct sum.

Next, take $l=2$ and let $|\underline{x}|^{2} P+\langle\underline{x}, \underline{u}\rangle Q+|\underline{u}|^{2} R+(\underline{x} \wedge \underline{u}) S=0$ for $P, Q, R, S \in L_{0}$. Obviously $P_{H}[(\underline{x} \wedge \underline{u}) S]=0$ and thus $S=0$; from the harmonic Fischer decomposition it then follows that the remaining $P, Q, R$ also have to be zero. Thus

$$
L_{2} \simeq|\underline{x}|^{2} L_{0} \oplus\langle\underline{x}, \underline{u}\rangle L_{0} \oplus|\underline{u}|^{2} L_{0} \oplus(\underline{x} \wedge \underline{u}) L_{0}
$$

For the general case $l>2$, we distinguish between $l$ odd or even. By the isomorphism (2):

$$
\begin{aligned}
\mathcal{R}_{2 j+1}(\underline{x}, \underline{u}) & \simeq \mathcal{I}_{2 j} \otimes(\underline{x} \oplus \underline{u}) \\
\mathcal{R}_{2 j}(\underline{x}, \underline{u}) & \left.\simeq \mathcal{I}_{2 j} \otimes 1 \oplus \mathcal{I}_{2 j-2} \otimes(\underline{x} \wedge \underline{u})\right) \\
& \simeq \mathcal{I}_{2 j-2} \otimes\left(|\underline{x}|^{2} \oplus\langle\underline{x}, \underline{u}\rangle \oplus|\underline{u}|^{2} \oplus(\underline{x} \wedge \underline{u})\right) .
\end{aligned}
$$

Take $l$ odd, $L_{2 j+1}=\mathfrak{f}_{-}^{2 j+1} L_{0} \simeq \mathcal{R}_{2 j+1}(\underline{x}, \underline{u}) \cdot L_{0} \simeq \mathcal{I}_{2 j} \cdot L_{1}$. The condition $\mathfrak{f}_{+}^{2} L_{1}=0$ implies $\mathfrak{p}_{+} L_{1}=0$, hence $L_{1}$ is a subspace of $\mathcal{H}$. By the harmonic Fischer decomposition $\mathcal{I}_{2 j} \cdot L_{1} \simeq$ $\mathcal{I}_{2 j} \otimes L_{1}$. Since $L_{1} \simeq(\underline{x} \oplus \underline{u}) \otimes L_{0}$ we have $L_{2 j+1} \simeq \mathcal{R}_{2 j+1}(\underline{x}, \underline{u}) \otimes L_{0}$.

For $l$ even, $L_{2 j}=\mathfrak{f}_{-}^{2 j} L_{0} \simeq \mathcal{R}_{2 j}(\underline{x}, \underline{u}) \cdot L_{0} \simeq \mathcal{I}_{2 j} \cdot L_{0}+\mathcal{I}_{2 j-2} \cdot(\underline{x} \wedge \underline{u}) L_{0}$. Let $\left\{J_{2 j, \alpha}, \alpha \in A_{2 j}\right\}$ be a basis of $\mathcal{I}_{2 j}$. and let $\left\{M_{\beta}, \beta \in B\right\}$ be a basis of $\mathcal{M}$. Suppose

$$
\sum_{\alpha \beta} J_{2 j, \alpha} M_{\beta}+\sum_{\lambda \beta} J_{2 j-2, \lambda}(\underline{x} \wedge \underline{u}) M_{\beta}=0 .
$$

The second sum can be written as

$$
\sum_{\lambda \beta} J_{2 j-2, \lambda}\left(P_{H}\left[(\underline{x} \wedge \underline{u}) M_{\beta}\right]+Q_{\beta}\right)=0
$$

with $Q_{\beta} \in \mathfrak{p}_{-} \mathcal{H}$ and thus $\sum_{\lambda \beta} J_{2 j-2, \lambda} Q_{\beta} \in \mathfrak{p}_{-}^{j} \mathcal{H}$. Therefore the right hand side of (17) is of the form

$$
\mathfrak{p}_{-}^{j} \mathcal{H}+\sum_{\lambda \beta} J_{2 j-2, \lambda} P_{H}\left[(\underline{x} \wedge \underline{u}) M_{\beta}\right]=0 .
$$

Since $\mathfrak{p}_{-}^{j-1} \mathcal{H} \perp \mathfrak{p}_{-}^{j} \mathcal{H}$, we have $\sum_{\lambda \beta} J_{2 j-2, \lambda} P_{H}\left[(\underline{x} \wedge \underline{u}) M_{\beta}\right]=0$. By construction, $J_{2 j-2, \lambda}$ are linearly independent invariants; thus by the harmonic Fischer decomposition $P_{H}\left[(\underline{x} \wedge \underline{u}) M_{\beta}\right]=$ 0 or $M_{\beta}=0$ for all $\beta$. Hence $\mathcal{R}_{2 j}(\underline{x}, \underline{u}) \cdot L_{0} \simeq \mathcal{R}_{2 j}(\underline{x}, \underline{u}) \otimes L_{0}$. 


\section{$6 \quad$ The Fischer decomposition in the non-stable range $m=3$}

We will now give an alternative easy proof of the monogenic Fischer decomposition which is based on a dimension computation (cf. lemma 10). This argument provides some extra information; it shows that the usual stable range $m \geq 4$ can be replaced by the milder condition $m \geq 3$. We will also show that theorem 6 and the harmonic Fischer decomposition (theorem 3) remain valid if $m=3$. Although we only need to prove the statements for $m=3$, we have chosen, for reasons of clarity, to reformulate this theorems under this new assumption $m \geq 3$. This is also motivated by our proofs in this section; all of them rely on the global condition $m \geq 3$. It turns out that this condition is now also sharp. This will be demonstrated by constructing counterexamples to the Fischer decomposition for $m=2$.

Theorem 12 (Monogenic Fischer decomposition: alternative proof) Let $m \geq 3$ and let $V=\mathbb{C}_{m}$ or $\mathbb{S}$. Then:

$$
\mathcal{P}\left(\mathbb{R}^{m \times 2}, V\right) \simeq \mathcal{R}(\underline{x}, \underline{u}) \otimes \mathcal{M}\left(\mathbb{R}^{m \times 2}, V\right) .
$$

Proof.

We will prove the statement for the subspace $\mathcal{P}_{k, l}\left(\mathbb{R}^{m \times 2}, V\right)$ of bidegree $(k, l)$. By $(5)$ :

$$
\mathcal{P}\left(\mathbb{R}^{m \times 2}, V\right)=\mathcal{R}(\underline{x}, \underline{u}) \cdot \mathcal{M}\left(\mathbb{R}^{m \times 2}, V\right) .
$$

Let $\mathcal{I}_{p, q}(\mathrm{SO}(m))$ and $\mathcal{R}_{p, q}(\underline{x}, \underline{u})$ be the subspaces of polynomial invariants of bidegree $(p, q)$. If we put $\mathcal{I}_{p, q}:=\mathcal{I}_{p, q}(\mathrm{SO}(m))$, we obtain by lemma 1 :

$$
\mathcal{R}_{p, q}(\underline{x}, \underline{u})=\mathcal{I}_{p, q} \oplus \underline{x} \wedge \underline{u} \mathcal{I}_{p-1, q-1} \oplus \underline{x} \mathcal{I}_{p-1, q} \oplus \underline{u} \mathcal{I}_{p, q-1} .
$$

Hence

$$
\begin{aligned}
\mathcal{P}_{k, l}= & \sum_{p=0}^{k} \sum_{q=0}^{l} \mathcal{I}_{p, q} \cdot \mathcal{M}_{k-p, l-q}+\underline{x} \wedge \underline{u} \sum_{p=1}^{k} \sum_{q=1}^{l} \mathcal{I}_{p-1, q-1} \cdot \mathcal{M}_{k-p, l-q} \\
& +\underline{x} \sum_{p=1}^{k} \sum_{q=0}^{l} \mathcal{I}_{p-1, q} \cdot \mathcal{M}_{k-p, l-q}+\underline{u} \sum_{p=0}^{k} \sum_{q=1}^{l} \mathcal{I}_{p, q-1} \cdot \mathcal{M}_{k-p, l-q} \\
= & (\mathrm{I})+(\mathrm{II})+(\mathrm{III})+(\mathrm{IV}) .
\end{aligned}
$$

A basis for the vector space $\mathcal{I}_{p, q}$ follows from the isomorphism: $\mathcal{I}(\mathrm{SO}(m)) \simeq \mathcal{P}\left(|\underline{x}|^{2},\langle\underline{x}, \underline{u}\rangle,|\underline{u}|^{2}\right)$. Put $r=\min (k-2 i, l-2 s)$. The summand (I) can be rewritten as

$$
\sum_{i=0}^{\left[\frac{k}{2}\right]} \sum_{s=0}^{\left[\frac{l}{2}\right]} \sum_{j=0}^{r}|\underline{x}|^{2 i}|\underline{u}|^{2 s}\langle\underline{x}, \underline{u}\rangle^{j} \mathcal{M}_{k-2 i-j, l-2 s-j} .
$$

In this proof, primes in the notation for polynomial spaces mean polynomial spaces on $\mathbb{R}^{m-1}$, so e.g. $\mathcal{P}_{k}^{\prime}:=\mathcal{P}_{k}\left(\mathbb{R}^{m-1}, V\right)$. According to the dimension formula of lemma 10 :

$$
\operatorname{dim} \mathcal{M}_{k, l}=\operatorname{dim} \mathcal{P}_{k, l}^{\prime}-\operatorname{dim} \mathcal{P}_{k-1, l-1}^{\prime}
$$

As a result, the following finite sum is telescoping and reduces to:

$$
\begin{aligned}
\sum_{j=0}^{r} \operatorname{dim}\left(\langle\underline{x}, \underline{u}\rangle^{j} \mathcal{M}_{k-2 i-j, l-2 s-j}\right) & =\sum_{j=0}^{r}\left(\operatorname{dim} \mathcal{P}_{k-2 i-j, l-2 s-j}^{\prime}-\operatorname{dim} \mathcal{P}_{k-1-2 i-j, l-1-2 s-j}^{\prime}\right) \\
& =\operatorname{dim} \mathcal{P}_{k-2 i, l-2 s}^{\prime} .
\end{aligned}
$$


This yields for the sums of the dimensions of the spaces occurring in the four summands:

$$
\begin{aligned}
& \text { (I) : } \sum_{i=0}^{\left[\frac{k}{2}\right]} \sum_{s=0}^{\left[\frac{l}{2}\right]} \operatorname{dim} \mathcal{P}_{k-2 i, l-2 s}^{\prime} \quad \text { (II) }: \sum_{i=0}^{\left[\frac{k-1}{2}\right]} \sum_{s=0}^{\left[\frac{l-1}{2}\right]} \operatorname{dim} \mathcal{P}_{k-1-2 i, l-1-2 s}^{\prime} \\
& \text { (III) : } \quad \sum_{i=0}^{\left[\frac{k-1}{2}\right]} \sum_{s=0}^{\left[\frac{l}{2}\right]} \operatorname{dim} \mathcal{P}_{k-1-2 i, l-2 s}^{\prime} \quad \text { (IV) }: \sum_{i=0}^{\left[\frac{k}{2}\right]} \sum_{s=0}^{\left[\frac{l-1}{2}\right]} \operatorname{dim} \mathcal{P}_{k-2 i, l-2 s-1}^{\prime} .
\end{aligned}
$$

The total sum of this four pieces can now be factorized as

$$
\left(\sum_{i=0}^{\left[\frac{k}{2}\right]} \operatorname{dim} \mathcal{P}_{k-2 i}^{\prime}+\sum_{i=0}^{\left[\frac{k-1}{2}\right]} \operatorname{dim} \mathcal{P}_{k-1-2 i}^{\prime}\right)\left(\sum_{s=0}^{\left[\frac{l}{2}\right]} \operatorname{dim} \mathcal{P}_{l-2 s}^{\prime}+\sum_{s=0}^{\left[\frac{l-1}{2}\right]} \operatorname{dim} \mathcal{P}_{l-1-2 s}^{\prime}\right)
$$

and equals $\operatorname{dim} \mathcal{P}_{k} \operatorname{dim} \mathcal{P}_{l}=\operatorname{dim} \mathcal{P}_{k, l}$. Therefore, with respect to our choice of basis for $\mathcal{R}(\underline{x}, \underline{u})$, the expression (18) is necessarily a direct sum and:

$$
\mathcal{R}(\underline{x}, \underline{u}) \cdot \mathcal{M}\left(\mathbb{R}^{m \times 2}, V\right) \simeq \mathcal{R}(\underline{x}, \underline{u}) \otimes \mathcal{M}\left(\mathbb{R}^{m \times 2}, V\right) .
$$

We can now extend the harmonic Fischer decomposition in theorem 3 to $m=3$.

Theorem 13 (Harmonic Fischer decomposition in two vector variables for $m \geq 3$ ) :

$$
\mathcal{P}\left(\mathbb{R}^{m \times 2}, \mathbb{C}\right) \simeq \mathcal{I}(\mathrm{SO}(m), 2) \otimes \mathcal{H}\left(\mathbb{R}^{m \times 2}, \mathbb{C}\right) .
$$

Proof.

By the primitive Fischer harmonic decomposition (which is in fact valid for $m \geq 2$ ):

$$
\mathcal{P}\left(\mathbb{R}^{m \times 2}, V\right)=\bigoplus_{j \geq 0} \mathfrak{p}_{-}^{j} \mathcal{H}\left(\mathbb{R}^{m \times 2}, V\right) \simeq \bigoplus_{j \geq 0} \mathcal{I}_{2 j}(\mathrm{SO}(m)) \cdot \mathcal{H}\left(\mathbb{R}^{m \times 2}, V\right)
$$

Let $\left\{J_{2 j, \alpha}, \alpha \in A_{2 j}\right\}$ be a basis of $\mathcal{I}_{2 j}(\mathrm{SO}(m))$ and suppose that $\sum_{\alpha} J_{2 j, \alpha} H_{\alpha}=0, H_{\alpha} \in$ $\mathcal{H}\left(\mathbb{R}^{m \times 2}, V\right)$. Decompose the harmonics $H_{\alpha}$ in monogenics:

$$
H_{\alpha}=M_{\alpha}^{0,0}+\left(\underline{x} M_{\alpha}^{1,0}+\underline{u} M_{\alpha}^{0,1}\right)+P_{H}\left[(\underline{x} \wedge \underline{u}) M_{\alpha}^{1,1}\right] \in \mathcal{M} \oplus \mathfrak{f}_{-} \mathcal{M} \oplus \mathfrak{f}_{-}^{2} \mathcal{M} .
$$

Notice that $J_{2 j, \alpha}$ acts as an element of $\mathfrak{f}_{-}^{2 j}$, hence

$$
\sum_{\alpha} J_{2 j, \alpha} H_{\alpha} \in \mathfrak{f}_{-}^{2 j} \mathcal{M} \oplus \mathfrak{f}_{-}^{2 j+1} \mathcal{M} \oplus \mathfrak{f}_{-}^{2 j+2} \mathcal{M}
$$

and the condition $\sum_{\alpha} J_{2 j, \alpha} H_{\alpha}=0$ implies

$$
\sum_{\alpha} J_{2 j, \alpha} M_{\alpha}^{0,0}=\sum_{\alpha} J_{2 j, \alpha}\left(\underline{x} M_{\alpha}^{1,0}+\underline{u} M_{\alpha}^{0,1}\right)=\sum_{\alpha} J_{2 j, \alpha} P_{H}\left[(\underline{x} \wedge \underline{u}) M_{\alpha}^{1,1}\right]=0 .
$$

Recall that by (2) the $\operatorname{Spin}(m)$-invariant polynomials $J_{2 j, \alpha} \underline{x}$ and $J_{2 j, \alpha} \underline{u}, \alpha \in A_{2 j}$ define a basis of $\mathfrak{f}_{-}^{2 j+1}$. Applying the monogenic Fischer decomposition for $m \geq 3$ (theorem 12), we obtain 
from the first two equalities that $M_{\alpha}^{0,0}=M_{\alpha}^{1,0}=M_{\alpha}^{0,1}=0$, for all $\alpha$. The third equality shows that

$$
\sum_{\alpha} J_{2 j, \alpha}\left((\underline{x} \wedge \underline{u}) M_{\alpha}^{1,1}+\frac{1}{E_{x}+E_{u}+m-4} T_{1,1} M_{\alpha}^{1,1}\right)
$$

must be zero. In this case the $\operatorname{Spin}(m)$-invariant polynomials $J_{2 j, \alpha}|\underline{x}|^{2}, J_{2 j, \alpha}|\underline{u}|^{2}, J_{2 j, \alpha}\langle\underline{x}, \underline{u}\rangle$ and $J_{2 j, \alpha} \underline{x} \wedge \underline{u}, \alpha \in A_{2 j}$ define a basis of $\mathfrak{f}_{-}^{2 j+2}$, hence $M_{\alpha}^{1,1}=0$ and thus $H_{\alpha}=0$ for all $\alpha$.

\section{$7 \quad$ Counterexamples for $m=2$}

We now explain how to generate counterexamples to the monogenic and harmonic Fischer decomposition in two vector variables if $m=2$. These examples illustrate that if the dimension of the space $\mathbb{R}^{m}$ is too small (here $m=2$ ), the space of polynomials $\mathcal{P}\left(\mathbb{R}^{2 \times 2}, V\right.$ ) is not a free module over either the algebra of invariants $\mathcal{R}(\underline{x}, \underline{u})$ or $\mathcal{I}_{2}(\mathrm{SO}(2))$.

Let $\underline{x}, \underline{u}, \underline{\tau} \in \mathbb{C}_{m}^{1}$. Consider the splitting of $\underline{\tau}(\underline{x} \wedge \underline{u})$ in its 1- and 3-vector part:

$$
\underline{\tau}(\underline{x} \wedge \underline{u})=\langle\underline{x}, \underline{\tau}\rangle \underline{u}-\langle\underline{u}, \underline{\tau}\rangle \underline{x}+\underline{\tau} \wedge \underline{x} \wedge \underline{u} .
$$

Choose $\underline{\tau}=e_{1}+i e_{2}$, then $\underline{\tau}$ is a null vector: $\underline{\tau}^{2}=0$. Let moreover $m=2$; then $\underline{\tau} \wedge \underline{x} \wedge \underline{u}=0$. Hence, after taking the reversed order of identity (20) in $\mathbb{C}_{2}$ we have:

$$
(\underline{x} \wedge \underline{u}) \tau=-\underline{u}\langle\underline{x}, \underline{\tau}\rangle+\underline{x}\langle\underline{u}, \underline{\tau}\rangle
$$

and after right multiplication with $\underline{\tau}$ we obtain (because of $\underline{\tau}^{2}=0$ ):

$$
-\underline{u}\langle\underline{x}, \underline{\tau}\rangle \underline{\tau}+\underline{x}\langle\underline{u}, \underline{\tau}\rangle \underline{\tau}=0 .
$$

Clearly $M_{1}=-\langle\underline{u}, \underline{\tau}\rangle \underline{\tau}, M_{2}=\langle\underline{x}, \underline{\tau}\rangle \underline{\tau}$ belong to $\mathcal{M}\left(\mathbb{R}^{2 \times 2}, \mathbb{C}_{2}\right)$, thus giving rise to a non-trivial identity between $\operatorname{Spin}(2)$-invariants and monogenics:

$$
\underline{x} M_{1}+\underline{u} M_{2}=0 .
$$

This identity shows that $\mathcal{R}_{1}(\underline{x}, \underline{u}) \cdot \mathcal{M}\left(\mathbb{R}^{2 \times 2}, \mathbb{C}_{2}\right)$ is not isomorphic with $\mathcal{R}_{1}(\underline{x}, \underline{u}) \otimes \mathcal{M}\left(\mathbb{R}^{2 \times 2}, \mathbb{C}_{2}\right)$, i.e. the map $\mathcal{R}_{1}(\underline{x}, \underline{u}) \otimes \mathcal{M}\left(\mathbb{R}^{2 \times 2}, \mathbb{C}_{2}\right) \rightarrow \mathcal{R}_{1}(\underline{x}, \underline{u}) \cdot \mathcal{M}\left(\mathbb{R}^{2 \times 2}, \mathbb{C}_{2}\right): J \otimes M \mapsto J M$ is not injective. For the harmonic case we proceed in the same way. Expressing $(\underline{\tau} \wedge \underline{x} \wedge \underline{u})^{2}$ in terms of the determinant of the Gramian matrix of the vectors $\underline{x}, \underline{u}, \underline{\tau}$ :

$$
(\underline{\tau} \wedge \underline{x} \wedge \underline{u})^{2}=\left|\begin{array}{ccc}
\langle\underline{x}, \underline{x}\rangle & \langle\underline{x}, \underline{u}\rangle & \langle\underline{x}, \underline{\tau}\rangle \\
\langle\underline{u}, \underline{x}\rangle & \langle\underline{u}, \underline{u}\rangle & \langle\underline{u}, \underline{\tau}\rangle \\
\langle\underline{\tau}, \underline{x}\rangle & \langle\underline{\tau}, \underline{u}\rangle & \langle\underline{\tau}, \underline{\tau}\rangle
\end{array}\right|
$$

we obtain the following identity in $\mathcal{P}\left(\mathbb{R}^{2 \times 2}, \mathbb{C}\right)$ :

$$
2\langle\underline{x}, \underline{u}\rangle\langle\underline{x}, \underline{\tau}\rangle\langle\underline{u}, \underline{\tau}\rangle-|\underline{x}|^{2}\langle\underline{u}, \underline{\tau}\rangle^{2}-|\underline{u}|^{2}\langle\underline{x}, \underline{\tau}\rangle^{2}=0 .
$$

Here $H_{1}=2\langle\underline{x}, \underline{\tau}\rangle\langle\underline{u}, \underline{\tau}\rangle, H_{2}=-\langle\underline{u}, \underline{\tau}\rangle^{2}$ and $H_{3}=-\langle\underline{x}, \underline{\tau}\rangle^{2}$ belong to $\mathcal{H}\left(\mathbb{R}^{2 \times 2}, \mathbb{C}\right)$, thus providing examples of harmonic polynomials such that

$$
\langle\underline{x}, \underline{u}\rangle H_{1}+|\underline{x}|^{2} H_{2}+|\underline{u}|^{2} H_{3}=0 .
$$

This shows that $\mathcal{I}_{2}(\mathrm{SO}(2)) \cdot \mathcal{H}\left(\mathbb{R}^{2 \times 2}, V\right)$ is not isomorphic with $\mathcal{I}_{2}(\mathrm{SO}(2)) \otimes \mathcal{H}\left(\mathbb{R}^{2 \times 2}, V\right)$.

The same idea can be used to construct counterexamples for the harmonic and monogenic Fischer decomposition for $k(>2)$ vector variables in $\mathbb{R}^{m}$ where the number of vector variables is too big: i.e. $k>\left[\frac{m+1}{2}\right]$. 


\section{References}

[1] F. Brackx, H. De Schepper, D. Eelbode, V. Souček, The Howe dual pair in Hermitean Clifford analysis, Rev. Mat. Iberoamericana Volume 26, Number 2 (2010), 449-479.

[2] F. Brackx, R. Delanghe and F. Sommen, Clifford Analysis, Pitman, London, 1982.

[3] F. Brackx, D. Eelbode, T. Raeymaekers, L. Van de Voorde, Triple monogenic functions and higher spin Dirac operators, submitted.

[4] F. Brackx, D. Eelbode, L. Van de Voorde, Higher spin Dirac operators between spaces of simplicial monogenics in two vector variables, submitted.

[5] J. Bureš, F. Sommen, V. Souček, P. Van Lancker, Rarita-Schwinger type operators in Clifford analysis, Journal of Funct. Anal. 185, 2001, 425-456.

[6] J. Bureš, F. Sommen, V. Souček, P. Van Lancker, Symmetric analogues of RaritaSchwinger equations, Ann. Glob. Anal. Geom. 21 No. 3 (2001), 215-240.

[7] J. Bureš, F. Sommen, V. Souček, P. Van Lancker, Separation of variables in Clifford analysis and its application to Rarita-Schwinger field, In: T.E. Simos, G. Psihoyios, Ch. Tsitouras, Extended abstracts of the ICNAAM 2006 Conference, Official Conference of the European Society of Computational Methods in Sciences and Engineering, Crete, Greece, 2006, 630-633.

[8] F. Colombo, I. Sabadini, F. Sommen, D. C. Struppa, Analysis of Dirac Systems and Computational Algebra, Progress in Mathematical Physics, Vol. 39, Birkhäuser, 2004.

[9] D. Constales, The relative position of $L_{2}$-domains in complex and Clifford analysis, Ph. D. thesis, State Univ. Ghent, 1989-1990.

[10] O. Debarre, T. Ton-That, Representations of $S O(k, \mathbb{C})$ on Harmonic Polynomials on a Null cone, Proc. Am. Math. Soc., Vol. 112, No. 1, May 1991, 31-44.

[11] R. Delanghe, R. Lavicka, V. Soucek, The Fischer decomposition for Hodge-de Rham systems in Euclidean spaces, arXiv:1012.4994v1.

[12] R. Delanghe, F. Sommen, V. Souček, Clifford analysis and spinor valued functions, Kluwer Acad. Publ., Dordrecht, 1992.

[13] D. Eelbode, D. Smid, L. Van de Voorde, A note on polynomial solutions for higher spin Dirac operators, in preparation.

[14] J. Gilbert and M. Murray, Clifford algebras and Dirac operators in harmonic analysis, Cambridge University Press, 1991.

[15] R. Goodman, Multiplicity-Free Spaces and Schur-Weyl-Howe Duality in Representations of Real and P-Adic Groups (Tan Eng-Chye and Zhu Chen-Bo, Ed.), World Scientific Publishing Company, 2004-06.

[16] K. Gürlebeck, W. Sprössig, Quaternionic and Clifford Calculus for Physicists and Engineers, J. Wiley \& Sons, Chichester, 1997. 
[17] S. Helgason, Invariants and fundamental functions, Acta Math. 109, 1963, 241-258.

[18] R. Howe, Dual Pairs in Physics: Harmonic Oscillators, Photons, Electrons and Singletons, Lect. Appl. Math. 21, Am. Math. Soc., 1985.

[19] R. Howe, E-C. Tan, J. Willenbring, Reciprocity Algebras and Branching for Classical Symmetric Pairs, Groups and analysis, London Math. Soc. Lecture Note Ser., 354, Cambridge Univ. Press, Cambridge, 2008, 191-231.

[20] M. Kashiwara, M. Vergne, On the Segal-Shale-Weil representations and harmonic polynomials, Inventiones Math. 44, 1978, 1-47.

[21] B. Kostant, Lie Group Representations on Polynomial Rings, Amer. J. Math., 85, 1963, 327-404.

[22] I. Sabadini, F. Sommen, D .C. Struppa, P. Van Lancker, Complexes of Dirac operators in Clifford algebras, Math. Zeit., 239(2), 2002, 293-320.

[23] F. Sommen, Clifford analysis in two and several vector variables, Appl. Anal. 73, 1999, 225253 .

[24] F. Sommen, Functions on the spin group, Advances in Applied Clifford algebras 6, No.1, 1996, 37-48.

[25] F. Sommen, An algebra of abstract vector variables, Portugal. Math. 54(3), 1997, 287-310.

[26] F. Sommen, N. Van Acker, SO(m)-invariant differential operators on Clifford algebra valued functions, Found. Phys. 23(11), 1993, 1491-1519.

[27] F. Sommen, N. Van Acker, Monogenic differential operators, Results in Math. 22(3-4), 1992, 781-798.

[28] F. Sommen, N. Van Acker, Functions of two vector variables, Adv. Appl. Clifford Algebras 4(1), 1994, 65-72.

[29] F. Sommen, N. Van Acker, Invariant differential operators on polynomial-valued functions, In: Clifford Algebras and their Applications in Mathematical Physics, Fund. Theories Phys. 55, Kluwer Academic Publishers, Dordrecht, 1993, 203-212.

[30] E. W. Stein, G. Weiss, Generalization of the Cauchy-Riemann equations and representations of the rotation group, Amer. J. Math. 90, 1968, 163-196.

[31] P. Van Lancker, F. Sommen, D. Constales, Models for irreducible representations of $\operatorname{Spin}(m)$, Advances in Applied Clifford Algebras, 11(S1), 2001, 271-289.

[32] H. Weyl, The classical groups, Their invariants and representations, Princeton Landmarks in Mathematics, Princeton University Press, Princeton, NJ, 1997. 